ESAIM: PROCEEDINGS, December 2008, Vol. 25, p. 91-113

E. Cancès, S. Faure, B. Graille, Editors

\title{
A NOVEL SECOND ORDER ACCURATE HYBRID NUMERICAL APPROACH FOR CONSERVATION LAWS
}

\author{
PASCAL JAISSON ${ }^{1,2}$ AND Florian De VUYST ${ }^{2,3,4}$
}

\begin{abstract}
In this paper, we present a novel one-parameter hybrid scheme for hyperbolic systems of conservation laws. The parameter value can be adapted in each numerical cell in order to obtain the advantages of Lax-Wendroff flux (second order scheme) where the solution is locally smooth. On the other hand, the scheme can switch to the Lax-Friedrichs one if necessary in order to be oscillation free (Total Variation Diminushing). Various numerical examples illustrate the efficiency of our scheme.

Résumé. Nous proposons dans cet article un nouveau schéma hybride à un paramètre pour les systèmes hyperboliques de lois de conservation. Le paramètre permet d'obtenir les bonnes propriétés du schéma de Lax-Wendroff lorsque la solution est régulière (schéma du second ordre) et de respecter la propriété de "Total Variation Diminushing" dans chaque cellule en utilisant si nécessaire le flux de Lax-Friedrichs. Nous présentons quelques expériences numériques illustrant l'efficacité de la méthode.
\end{abstract}

\section{INTRODUCTION}

We are interested in the initial value problems of systems of conservation law:

$$
\begin{aligned}
& \partial_{t} \mathbf{u}+\partial_{x} \mathbf{f}(\mathbf{u})=0, x \in \mathbb{R}, t \geq 0 \\
& \mathbf{u}(x, 0)=\mathbf{u}^{0}(x)
\end{aligned}
$$

We assume that $\mathbf{u}$ belongs to an open set $\Omega$ of $\mathbb{R}^{p}$. The physical flux $\mathbf{f}: \Omega \rightarrow \mathbb{R}^{p}$ is supposed to be locally Lipschitz continuous. The hyperbolic assumption means that for all states $\mathbf{u}$ in $\Omega$, the jacobian matrix $\mathbf{A}(\mathbf{u})$ of the flux:

$$
\mathbf{A}(\mathbf{u})=\partial_{\mathbf{u}} \mathbf{f}(\mathbf{u}) \in \mathcal{M}\left(\mathbb{R}^{p}\right)
$$

is diagonalisable in $\mathbb{R}$. To simplify, we assume that the system is strictly hyperbolic, i.e. for all $\mathbf{u} \in \Omega$ the multiplicity of each eigenvalue $\alpha_{k}(\mathbf{u})$ is one [6]. The eigenvalues are arranged in increasing order

$$
\alpha_{1}(\mathbf{u})<\alpha_{2}(\mathbf{u})<\ldots<\alpha_{p}(\mathbf{u}) .
$$

\footnotetext{
${ }^{1}$ CMAP, Ecole Polytechnique, INRIA Saclay-Ile-de-France, Equipe COMMANDS, Route de Saclay, 91128 Palaiseau cedex, FRANCE

${ }^{2}$ Laboratoire MAS, Ecole Centrale Paris, Grande voie des vignes 92295 Châtenay-Malabry cedex FRANCE

${ }^{3}$ CMLA, ENS Cachan, 94235 Cachan cedex, FRANCE

${ }^{4}$ Energy Conversion Research Center, Doshisha University, Kyotanabe campus, Kyoto, Japan
}

(C) EDP Sciences, SMAI 2008 
We want to find a numerical conservative scheme which is oscillation free (TVD) and as low diffusive as possible:

$$
\mathbf{u}_{j}^{n+1}=\mathbf{u}_{j}^{n}-\lambda^{n}\left(\phi\left(\mathbf{u}_{j}^{n}, \mathbf{u}_{j+1}^{n}, \lambda, \theta_{j+\frac{1}{2}}^{n}\right)-\phi\left(\mathbf{u}_{j-1}^{n}, \mathbf{u}_{j}^{n}, \lambda, \theta_{j-\frac{1}{2}}^{n}\right)\right)
$$

where $\lambda^{n}=\frac{\Delta t^{n}}{h}, \Delta t^{n}$ and $h$ are respectively the time and (constant) space steps. Our numerical hybrid flux may be written as:

$$
\phi(\mathbf{u}, \mathbf{v}, \lambda, \theta)=\frac{\mathbf{f}(\mathbf{u})+\mathbf{f}(\mathbf{v})}{2}-\frac{1}{2} \lambda^{\theta}|\overline{\mathbf{A}}(\mathbf{u}, \mathbf{v})|^{1+\theta}(\mathbf{v}-\mathbf{u})
$$

where the real parameter $\theta$ will depend on both time and space. The goal of the paper is to find a 'parameter' $\theta$ which guarantees the TVD property and the second order accuracy.

The paper is organized as follows. In section 1, the hybrid scheme is presented: definition of the numerical hybrid flux and equivalent equation. In section 2, we give conditions on $\theta$ to obtain a TVD scheme with second order accuracy in time and space for a nonlinear scalar conservation law. Then we heuristically extend our scheme to the case of systems of conservation laws. In section 3, some numerical experiments are presented.

\section{Numerical ANALYSis OF THE HYBRID SCHEME}

\subsection{Construction of the hybrid scheme}

Let us first recall some common classical fluxes: Lax-Wendroff, Lax-Friedrichs, Modified Lax-Friedrichs, Roe and Rusanov. We shall show that it is possible to interpolate three of these six fluxes thanks to a single parameter $\theta$. This will define our one-parameter family of hybrid flux.

\subsubsection{Construction of the hybrid numerical flux}

Let us consider a uniform spatial mesh with a constant space step $h$. We denote $x_{j}=j h$ the mesh points and $x_{j+\frac{1}{2}}=\left(j+\frac{1}{2}\right) h$ some interfaces. The time step $\Delta t^{n}$ can be variable and we set $t^{n+1}=t^{n}+\Delta t^{n}$. At time $t^{n}, \mathbf{u}_{j}^{n}, j \in \mathbb{Z}$ defines a discrete approximation of the continuous solution $\mathbf{u}$ :

$$
\mathbf{u}_{j}^{n} \approx \frac{1}{h} \int_{x_{j-1 / 2}}^{x_{j+1 / 2}} \mathbf{u}\left(x, t^{n}\right) d x .
$$

In the sequel, we denote

$$
\lambda=\frac{\Delta t^{n}}{h},
$$

forgetting the $n$-index for simplicity. The solutions of (1) are approximated by a conservative scheme:

$$
\mathbf{u}_{j}^{n+1}=\mathbf{u}_{j}^{n}-\lambda\left(\phi\left(\mathbf{u}_{j}^{n}, \mathbf{u}_{j+1}^{n}, \lambda\right)-\phi\left(\mathbf{u}_{j-1}^{n}, \mathbf{u}_{j}^{n}, \lambda\right)\right)
$$

where the numerical flux $\phi: \Omega \times \Omega \rightarrow \mathbb{R}^{p}$ is Lipschitz continuous on $\Omega \times \Omega$. Let $\mathbf{A}(\mathbf{u})=\mathbf{f}^{\prime}(\mathbf{u})$. We first recall some common numerical fluxes from the Finite Volume community. The Lax-Wendroff scheme (LW-scheme) [9] is second order accurate. Its numerical flux may be written in two forms: either

$$
\phi(\mathbf{u}, \mathbf{v})=\frac{\mathbf{f}(\mathbf{u})+\mathbf{f}(\mathbf{v})}{2}-\frac{1}{2} \lambda \overline{\mathbf{A}}(\mathbf{u}, \mathbf{v})(\mathbf{f}(\mathbf{v})-\mathbf{f}(\mathbf{u}))
$$

or

$$
\phi(\mathbf{u}, \mathbf{v})=\frac{\mathbf{f}(\mathbf{u})+\mathbf{f}(\mathbf{v})}{2}-\frac{1}{2} \lambda \overline{\mathbf{A}}(\mathbf{u}, \mathbf{v})^{2}(\mathbf{v}-\mathbf{u})
$$


where $\overline{\mathbf{A}}(\mathbf{u}, \mathbf{v})$ is a diagonalisable matrix with the consistency property $\overline{\mathbf{A}}(\mathbf{u}, \mathbf{u})=\mathbf{A}(\mathbf{u})$. Notice that if the mean matrix $\overline{\mathbf{A}}(\mathbf{u}, \mathbf{v})$ is chosen as a Roe matrix, i.e. with the additionnal constraint $\overline{\mathbf{A}}(\mathbf{u}, \mathbf{v})(\mathbf{v}-\mathbf{u})=\mathbf{f}(\mathbf{v})-\mathbf{f}(\mathbf{u})$ (if it exists), then the two forms (5) and (6) are equivalent. The form (5) belongs to the general family of characteristic flux scheme [4]. In the sequel, we will take advantage of the two forms. The LW scheme is conditionally linearly $L^{2}$-stable but also known to be dispersive and strongly oscillatory.

The flux of the Lax-Friedrichs scheme (LF-scheme) [2,8] is

$$
\phi(\mathbf{u}, \mathbf{v}, \lambda)=\frac{\mathbf{f}(\mathbf{u})+\mathbf{f}(\mathbf{v})}{2}-\frac{1}{2 \lambda}(\mathbf{v}-\mathbf{u})
$$

This first order accurate scheme is very stable but also known to be strongly dissipative.

The flux of the Modified Lax-Friedrichs scheme (LFM-scheme) [14] is

$$
\phi(\mathbf{u}, \mathbf{v}, \lambda)=\frac{\mathbf{f}(\mathbf{u})+\mathbf{f}(\mathbf{v})}{2}-\frac{1}{4 \lambda}(\mathbf{v}-\mathbf{u})
$$

This scheme is an entropy scheme under a CFL condition but is strongly dissipative too. Next, the Roe flux [10] is :

$$
\phi(\mathbf{u}, \mathbf{v}, \lambda)=\frac{\mathbf{f}(\mathbf{u})+\mathbf{f}(\mathbf{v})}{2}-\frac{1}{2}|\overline{\mathbf{A}}(\mathbf{u}, \mathbf{v})|(\mathbf{v}-\mathbf{u}),
$$

where $\overline{\mathbf{A}}(\mathbf{u}, \mathbf{v})$ is a Roe's matrix [6]. Here the absolute value of a diagonalisable matrix $\mathbf{A}=R \operatorname{diag}\left(\alpha_{1}, \ldots, \alpha_{p}\right) R^{-1}$ is defined by $|\mathbf{A}|=R \operatorname{diag}\left(\left|\alpha_{1}\right|, \ldots,\left|\alpha_{p}\right|\right) R^{-1}$. The Roe scheme is particularly appreciated for its ability to accurately capture low-moving contact discontinuities but it violates the discrete entropy property. Lastly, we give the Rusanov flux [11]:

$$
\phi(\mathbf{u}, \mathbf{v}, \lambda)=\frac{\mathbf{f}(\mathbf{u})+\mathbf{f}(\mathbf{v})}{2}-\frac{1}{2 \lambda} \alpha_{\max }(\overline{\mathbf{A}}(\mathbf{u}, \mathbf{v}))(\mathbf{v}-\mathbf{u})
$$

where $\alpha_{\max }(\overline{\mathbf{A}}(\mathbf{u}, \mathbf{v}))$ is the spectral radius of $\overline{\mathbf{A}}(\mathbf{u}, \mathbf{v})$.

The three schemes (Lax-Wendroff, Lax-Friedrichs, Roe) can all be written in the form

$$
\mathbf{u}_{j}^{n+1}=\mathbf{u}_{j}^{n}-\lambda\left(\phi\left(\mathbf{u}_{j}^{n}, \mathbf{u}_{j+1}^{n}, \lambda, \theta\right)-\phi\left(\mathbf{u}_{j-1}^{n}, \mathbf{u}_{j}^{n}, \lambda, \theta\right)\right)
$$

where

$$
\phi(\mathbf{u}, \mathbf{v}, \lambda, \theta)=\frac{\mathbf{f}(\mathbf{u})+\mathbf{f}(\mathbf{v})}{2}-\frac{1}{2} \lambda^{\theta}|\overline{\mathbf{A}}(\mathbf{u}, \mathbf{v})|^{1+\theta}(\mathbf{v}-\mathbf{u}) .
$$

We recognize the Lax-Wendroff flux for $\theta=1$, the Roe's flux for $\theta=0$ and the Lax-Friedrichs flux for $\theta=-1$. The parameter $\theta$ can be interpreted as an artificial viscosity controller. For $\theta=1$, the Lax-Wendroff scheme is second order accurate scheme in both space and time but generally violates the entropy property. The Lax-Friedrichs scheme is TVD for scalar conservation laws and fulfils a discrete entropy inequality, but it is not an accurate scheme. The Roe's scheme can violate the entropy property. Now, we want to combine the complementary advantages of these schemes. We then explore a new hybrid scheme where $\theta$ varies in an interval which contains $[-1,1]$. The hybrid scheme is:

$$
\mathbf{u}_{j}^{n+1}=\mathbf{u}_{j}^{n}-\lambda\left(\phi\left(\mathbf{u}_{j}^{n}, \mathbf{u}_{j+1}^{n}, \lambda, \theta_{j+\frac{1}{2}}^{n}\right)-\phi\left(\mathbf{u}_{j-1}^{n}, \mathbf{u}_{j}^{n}, \lambda, \theta_{j-\frac{1}{2}}^{n}\right)\right)
$$

with

and

$$
\phi(\mathbf{u}, \mathbf{v}, \lambda, \theta)=\frac{\mathbf{f}(\mathbf{u})+\mathbf{f}(\mathbf{v})}{2}-\frac{1}{2} \lambda^{\theta}|\overline{\mathbf{A}}(\mathbf{u}, \mathbf{v})|^{1+\theta}(\mathbf{v}-\mathbf{u})
$$

$$
\theta_{j+\frac{1}{2}}^{n}=\theta\left(\mathbf{u}_{j-1}^{n}, \mathbf{u}_{j}^{n}, \mathbf{u}_{j+1}^{n}, \mathbf{u}_{j+2}^{n}, \lambda\right)
$$




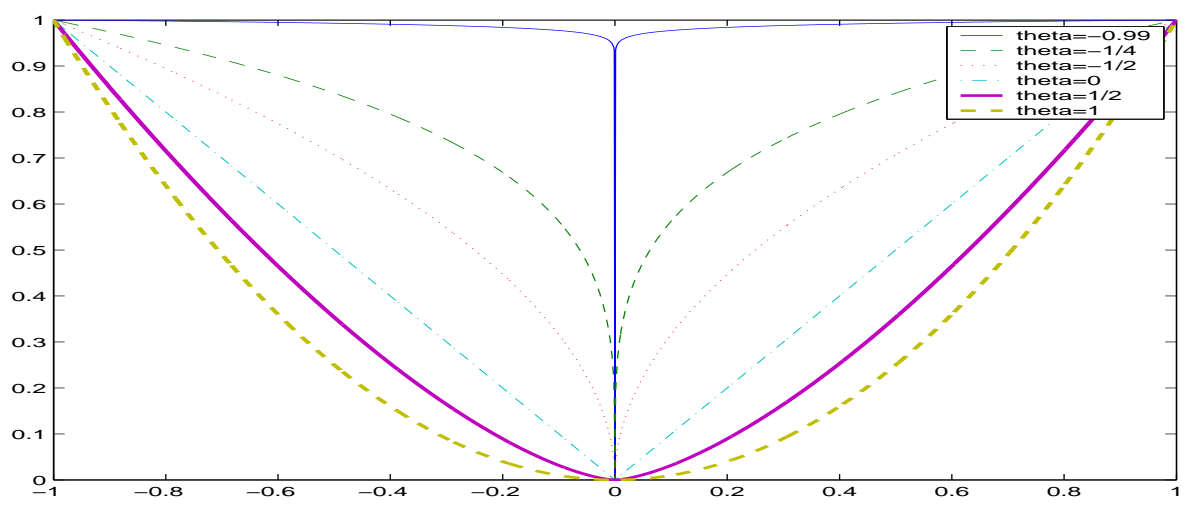

Figure 1. Function $x \mapsto|x|^{1+\theta}$ for different values of $\theta$.

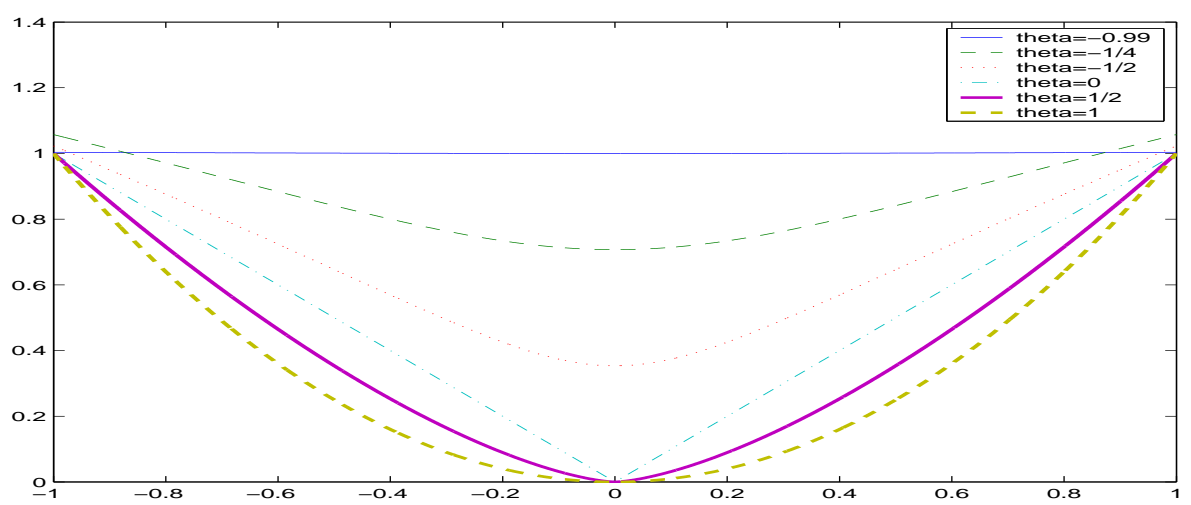

Figure 2. Function $x \mapsto\left(|x|^{2}+\min (0, \theta)^{2}\right)^{\frac{1+\theta}{2}}$ for different values of $\theta$.

Remark that due to the absolute value, the numerical flux is not regular at sonic point (zero eigenvalue for the jacobian A, [5]). But it is always possible to add a regularizing term to get Lipschitz continuity while preserving the LW-Roe-LF interpolation property. For example, the following numerical flux is a good candidate:

$$
\phi(\mathbf{u}, \mathbf{v}, \lambda, \theta)=\frac{\mathbf{f}(\mathbf{u})+\mathbf{f}(\mathbf{v})}{2}-\frac{1}{2} \lambda^{\theta}\left(|\overline{\mathbf{A}}(\mathbf{u}, \mathbf{v})|^{2}+\min (0, \theta)^{2} \mathbf{I}\right)^{\frac{1+\theta}{2}}(\mathbf{v}-\mathbf{u})
$$

Obviously, the numerical flux is locally Lipschitz continuous, uniformly in $\theta$ (even $C^{\infty}$ except for $\theta=0$ ). The figures 1 and 2 represent the functions $x \mapsto|x|^{1+\theta}$ and $x \mapsto\left(|x|^{2}+\min (0, \theta)^{2}\right)^{\frac{1+\theta}{2}}$ respectively for different values of $\theta \in[-1,1]$. Another possibility would be to use the Harten entropy fix which consists in regularizing the absolute value (see subsection 3.2.2). Anyway, the very local loss of regularity in the original flux does not seem to have spurious consequences at a numerical point of view (see the numerical experiments below). On one hand, we want to adapt $\theta$ in order to get local second order accuracy in the regions where the solution is smooth. On the other hand, the $\theta$ parameter is expected provide more numerical diffusion when the solution is no more smooth (discontinuities, strong variation...). Remark that the parameter $\theta$ can be negative in order to satisfy the entropy property. This will be analysed in a future work.

As example, below we give other instances of hybrid schemes.

LW-upwind-LF scheme: we may use the matrix $\overline{\mathbf{A}}(\mathbf{u}, \mathbf{v})=\partial_{\mathbf{u}} \mathbf{f}\left(\frac{\mathbf{u}+\mathbf{v}}{2}\right)$. The scheme is easier to implemente. Indeed, it is not necessary to compute a Roe matrix and the diagonalisation of $\partial_{\mathbf{u}} \mathbf{f}(\mathbf{u})$ is only necessary. 
LW-VFFC-LF scheme: we may use the VFFC scheme [3,4]. Indeed, we remark that the flux can be written

$$
\phi(\mathbf{u}, \mathbf{v}, \lambda, \theta)=\frac{\mathbf{f}(\mathbf{u})+\mathbf{f}(\mathbf{v})}{2}-\frac{1}{2} \operatorname{sign}(\overline{\mathbf{A}}) \lambda^{\theta}|\overline{\mathbf{A}}(\mathbf{u}, \mathbf{v})|^{\theta}(\mathbf{f}(\mathbf{v})-\mathbf{f}(\mathbf{u})),
$$

for $\theta \geq 0$ if $\overline{\mathbf{A}}$ is a Roe matrix. Here the sign of a diagonalisable matrix $\mathbf{A}=R \operatorname{diag}\left(\alpha_{1}, \ldots, \alpha_{p}\right) R^{-1}$ is defined as $\operatorname{sign}(\mathbf{A})=R \operatorname{diag}\left(\operatorname{sign}\left(\alpha_{1}\right), \ldots, \operatorname{sign}\left(\alpha_{p}\right)\right) R^{-1}$, where $\operatorname{sign}(x)$ is equal to 1 if $x>0,0$ if $x=0$ and -1 if $x<0$. So we set

$$
\phi(\mathbf{u}, \mathbf{v}, \lambda, \theta)=\frac{\mathbf{f}(\mathbf{u})+\mathbf{f}(\mathbf{v})}{2}-\frac{1}{2} \lambda^{\theta} \mathbf{B}(\mathbf{f}(\mathbf{v})-\mathbf{f}(\mathbf{u}))
$$

where $\mathbf{B}=\operatorname{sign}(\overline{\mathbf{A}})|\overline{\mathbf{A}}(\mathbf{u}, \mathbf{v})|^{\theta}$. The matrix $\overline{\mathbf{A}}$ needs only to verify $\overline{\mathbf{A}}(\mathbf{u}, \mathbf{u})=\partial_{\mathbf{u}} \mathbf{f}(\mathbf{u})$.

LW-Roe-Rusanov scheme: we may introduce other schemes. For example, we can consider the numerical flux

$$
\phi(\mathbf{u}, \mathbf{v}, \lambda, \theta)=\frac{\mathbf{f}(\mathbf{u})+\mathbf{f}(\mathbf{v})}{2}-\frac{1}{2} \lambda^{\theta^{+}} \alpha_{\max }^{\theta^{+}+1}\left|\frac{1}{\alpha_{\max }} \overline{\mathbf{A}}(\mathbf{u}, \mathbf{v})\right|^{1+\theta}(\mathbf{v}-\mathbf{u})
$$

where $\theta^{+}=\max (0, \theta)$. For this hybrid scheme, $\theta=1$ gives the Lax-Wendroff scheme, $\theta=0$ the Roe scheme and $\theta=-1$ the Rusanov scheme.

LW-Roe-LFM or LW-upwind-LFM scheme: lastly, we give a scheme where $\theta=1$ corresponds to the Lax-Wendroff scheme and $\theta=-1$ to the Modified Lax-Friedrichs scheme.

$$
\phi(\mathbf{u}, \mathbf{v}, \lambda, \theta)=\frac{\mathbf{f}(\mathbf{u})+\mathbf{f}(\mathbf{v})}{2}-\frac{1}{2} \lambda^{\theta} 2^{\theta^{-}}|\overline{\mathbf{A}}(\mathbf{u}, \mathbf{v})|^{1+\theta}(\mathbf{v}-\mathbf{u})
$$

where $\theta^{-}=\min (0, \theta)$. In this hybrid scheme, we obtain the Modified Lax-Friedrichs for $\theta=-1$ which is entropy satisfying. We will use the LW-Upwind-LFM scheme in our numerical experiment.

\subsection{Vector extension of hybrid schemes}

Our hybrid scheme introduces a scalar parameter $\theta$. In the case of systems of conservation laws, it is natural to look for a hybrid scheme with a vector parameter $\boldsymbol{\theta}=\left(\theta_{k}\right)_{k=1, \ldots, p}$. Let us suppose that the matrix $\overline{\mathbf{A}}(\mathbf{u}, \mathbf{v})$ is diagonalisable with:

$$
\overline{\mathbf{A}}(\mathbf{u}, \mathbf{v})=\mathbf{R}(\mathbf{u}, \mathbf{v}) \mathbf{D}(\mathbf{u}, \mathbf{v}) \mathbf{R}(\mathbf{u}, \mathbf{v})^{-1},
$$

where $\mathbf{D}(\mathbf{u}, \mathbf{v})=\operatorname{diag}\left(\alpha_{k}(\mathbf{u}, \mathbf{v})\right)$. We use the notation:

$$
|\overline{\mathbf{A}}(\mathbf{u}, \mathbf{v})|^{\boldsymbol{\theta}}=\mathbf{R}(\mathbf{u}, \mathbf{v}) \operatorname{diag}\left(\left|\alpha_{k}(\mathbf{u}, \mathbf{v})\right|^{\theta_{k}}\right) \mathbf{R}(\mathbf{u}, \mathbf{v})^{-1} .
$$

In this case, the numerical flux is

$$
\phi(\mathbf{u}, \mathbf{v}, \lambda, \boldsymbol{\theta})=\frac{\mathbf{f}(\mathbf{u})+\mathbf{f}(\mathbf{v})}{2}-\frac{1}{2}(\lambda \mathbf{I}){ }^{\boldsymbol{\theta}}|\overline{\mathbf{A}}(\mathbf{u}, \mathbf{v})|^{\mathbf{e}+\boldsymbol{\theta}}(\mathbf{v}-\mathbf{u})
$$

with $\mathbf{e}=(1, \ldots, 1)$; the matrix $\mathbf{I}$ is the identity matrix in $\mathbb{R}^{p}$.

Of course, we can rewrite all the other hybrid schemes presented above with the vector parameters $\boldsymbol{\theta}$.

\subsection{Equivalent equations for the hybrid scheme}

We shall study the influence of $\theta$ on the numerical diffusion in more detail. We give the equivalent equations associated to the scalar and the vector forms of the hybrid scheme respectively.

We write the second order Taylor expansion near the point $\left(x_{j}, t^{n}\right)$ in $(13),(14)$. We then obtain the equivalent 
PDE solved by the hybrid scheme at time-space second order accuracy. To simplify, let us suppose that $\theta$ is a locally constant function near the point $\left(x_{j}, t^{n}\right)$. It is easy to show that the equivalent equation of the scalar hybrid scheme is

$$
\partial_{t} \mathbf{u}+\partial_{x} \mathbf{f}(\mathbf{u})+\frac{\Delta t^{n}}{2} \partial_{x}\left[\mathbf{A}^{2}(\mathbf{u}) \partial_{x} \mathbf{u}\right]-\frac{1}{2}\left(\Delta t^{n}\right)^{\theta} h^{1-\theta} \partial_{x}\left[|\mathbf{A}(\mathbf{u})|^{1+\theta} \partial_{x} \mathbf{u}\right]=0
$$

Or, equivalently,

$$
\partial_{t} \mathbf{u}+\partial_{x} \mathbf{f}(\mathbf{u})-\frac{\Delta t^{n}}{2} \partial_{x}\left[\left(\lambda^{\theta-1}|\mathbf{A}(\mathbf{u})|^{\theta+1}-\mathbf{A}^{2}(\mathbf{u})\right) \partial_{x} \mathbf{u}\right]=0
$$

The conditions on the eigenvalues $\alpha_{k}$ of $\mathbf{A}(\mathbf{u})$ to have a positive diffusion matrix are:

$$
\left(\lambda\left|\alpha_{k}\right|\right)^{\theta-1}-1 \geq 0 \quad \forall k \in\{1, \ldots, p\}
$$

For $\theta \leq 1$, we retrieve the classical condition of Courant-Friedrichs-Lewy on the time step $\Delta t^{n}$ :

$$
\lambda\left|\alpha_{k}\right| \leq 1 \quad \forall k
$$

We remark that the diffusion matrix is exactly zero for $\theta=1$; indeed, the numerical scheme is in this case the Lax-Wendroff one. Otherwise, the numerical diffusion rate is governed by the eigenvalues of the diffusion matrix which are

$$
\left(\left(\lambda\left|\alpha_{k}\right|\right)^{\theta-1}-1\right)\left(\alpha_{k}\right)^{2} \text { if } \alpha_{k} \neq 0 \text { and } 0 \text { otherwise. }
$$

For $\lambda\left|\alpha^{k}\right|>0$, the function

is $\mathcal{C}^{\infty}$ on $[-1,+\infty)$. Moreover,

$$
q: \theta \mapsto\left(\alpha_{k}\right)^{2}\left(\left(\lambda\left|\alpha_{k}\right|\right)^{\theta-1}-1\right)
$$

$$
q^{\prime}(\theta)=\left(\alpha_{k}\right)^{2} \ln \left(\lambda\left|\alpha_{k}\right|\right)\left(\lambda\left|\alpha_{k}\right|\right)^{\theta-1}<0
$$

for all $\theta$, if the CFL condition (20) is satisfied. In particular that means that the diffusion rate is a decreasing function of the parameter $\theta$.

Remark 1.1. For $\theta \geq 1$, the function $q$ is negative and the limit of $q$ when $\theta$ tends to $+\infty$ is $-\left(\alpha_{k}\right)^{2}$. Thus, for $\theta \geq 1$ the matrix $\lambda^{\theta}|\mathbf{A}(\mathbf{u})|^{\theta+1}-\mathbf{A}^{2}(\mathbf{u})$ is an anti-diffusion matrix whose intensity remains bounded when $\theta$ tends to $+\infty$.

Let us emphasize that the calculus can be extended to the vector case without difficulty with the same conclusions:

$$
\partial_{t} \mathbf{u}+\partial_{x} \mathbf{f}(\mathbf{u})-\frac{\Delta t^{n}}{2} \partial_{x}\left[\left(\lambda^{\boldsymbol{\theta}-\mathbf{e}}|\mathbf{A}(\mathbf{u})|^{\mathbf{e}+\boldsymbol{\theta}}-\mathbf{A}^{2}(\mathbf{u})\right) \partial_{x} \mathbf{u}\right]=0 .
$$

The trade-off between accuracy and stability leads us to look for stability conditions and order of accuracy with respect to $\theta$.

\section{Determination of the FUnCtions $\theta$}

\subsection{Scalar linear transport equation}

As starting point, let us consider the linear case. We want to find the parameters $\theta_{j+\frac{1}{2}}^{n}$ that give the TVD property. Consider the equation

$$
\partial_{t} u+a \partial_{x} u=0
$$

where $a$ is constant. We may suppose $a>0$ for example. The hybrid scheme (12) in the scalar case is simply:

$$
u_{j}^{n+1}=u_{j}^{n}-\lambda\left(\phi_{j+\frac{1}{2}}^{n}-\phi_{j-\frac{1}{2}}^{n}\right)
$$


with the numerical flux

$$
\phi_{j+\frac{1}{2}}^{n}=a \frac{u_{j}^{n}+u_{j+1}^{n}}{2}-\frac{1}{2} \lambda^{\theta_{j+\frac{1}{2}}^{n}} a^{1+\theta_{j+\frac{1}{2}}^{n}}\left(u_{j+1}^{n}-u_{j}^{n}\right)
$$

2.1.1. TVD and stability analysis of the hybrid scheme with the Harten criteria

We want to determine some parameters $\theta_{j+\frac{1}{2}}^{n}$ such that the hybrid scheme has TVD and $L^{\infty}$ properties. Let us define

$$
\nu=a \lambda, \quad \Delta u_{j+\frac{1}{2}}=u_{j+1}^{n}-u_{j}^{n}
$$

and

$$
r_{j}=\frac{\Delta u_{j-\frac{1}{2}}}{\Delta u_{j+\frac{1}{2}}}
$$

Following ideas from Harten and Sweby [7,13], we write the numerical scheme:

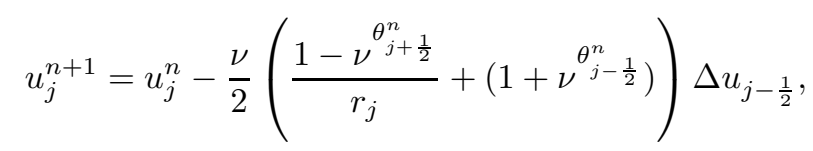

which gives in incremental form [6]

$$
u_{j}^{n+1}=u_{j}^{n}+C_{j+\frac{1}{2}}^{n} \Delta u_{j+\frac{1}{2}}-D_{j-\frac{1}{2}}^{n} \Delta u_{j-\frac{1}{2}}
$$

with the coefficients

$$
\left\{\begin{array}{l}
C_{j+\frac{1}{2}}^{n}=0 \\
D_{j-\frac{1}{2}}^{n}=\frac{\nu}{2}\left(\frac{1-\nu^{\theta^{n}+\frac{1}{2}}}{r_{j}}+\left(1+\nu^{\theta^{n}-\frac{1}{2}}\right)\right) .
\end{array}\right.
$$

According to Harten's criteria [7], the hybrid scheme is TVD if for all $n$ and $j$,

$$
\begin{aligned}
& C_{j+\frac{1}{2}}^{n} \geq 0, D_{j+\frac{1}{2}}^{n} \geq 0 \\
& C_{j+\frac{1}{2}}^{n}+D_{j+\frac{1}{2}}^{n} \leq 1
\end{aligned}
$$

and is $L^{\infty}$-stable if for all $n$ and $j$,

$$
\begin{aligned}
& C_{j+\frac{1}{2}}^{n} \geq 0, D_{j+\frac{1}{2}}^{n} \geq 0 \\
& C_{j+\frac{1}{2}}^{n}+D_{j-\frac{1}{2}}^{n} \leq 1
\end{aligned}
$$

In our case, the two criteria become $0 \leq D_{j+\frac{1}{2}}^{n} \leq 1$. We are looking for $\theta_{j+\frac{1}{2}}^{n}$ satisfying these inequalities. We now suppose that the CFL condition is satisfied: $\nu<1$. Thus the function $\theta$ must verify: for all $r, r^{\prime}$,

$$
0 \leq \frac{\nu}{2}\left(\frac{1-\nu^{\theta(r, \nu)}}{r}+1+\nu^{\theta\left(r^{\prime}, \nu\right)}\right) \leq 1
$$

that is

$$
-1 \leq \frac{1-\nu^{\theta(r, \nu)}}{r}+\nu^{\theta\left(r^{\prime}, \nu\right)} \leq \frac{2}{\nu}-1
$$


First, we consider the left inequality in $(27):-1 \leq \frac{1-\nu^{\theta(r, \nu)}}{r}+\nu^{\theta\left(r^{\prime}, \nu\right)}$. We consider a parameter $\alpha$ in $[0 ; 1]$. We are looking for conditions on $\theta$ such that

$$
\left\{\begin{array}{l}
\alpha \leq \nu^{\theta\left(r^{\prime}, \nu\right)} \\
-1-\alpha \leq \frac{1-\nu^{\theta(r, \nu)}}{r}
\end{array}\right.
$$

The first inequality $\alpha \leq \nu^{\theta\left(r^{\prime}, \nu\right)}$ is verified if and only if $\theta\left(r^{\prime}, \nu\right) \leq \frac{\ln \alpha}{\ln \nu}$. For the second inequality $-1-\alpha \leq$ $\frac{1-\nu^{\theta(r, \nu)}}{r}$, we have two cases $r \geq 0$ and $r<0$.

For the first case $r \geq 0$, the inequality is verified if and only if $\theta(r, \nu) \geq \frac{\ln (1+(1+\alpha) r)}{\ln \nu}$. For the second case $r<0$, if $r \leq \frac{-1}{1+\alpha}$, the inequality is always satisfied. If $\frac{-1}{1+\alpha}<r<0$, the inequality is satisfied if and only if $\theta(r, \nu) \leq \frac{\ln (1+(1+\alpha) r)}{\ln \nu}$. We remark that if $r \in\left[\frac{-1}{1+\alpha} ; \frac{-1+\alpha}{1+\alpha}\right]$, then $\frac{\ln (1+(1+\alpha) r)}{\ln \nu} \geq \frac{\ln \alpha}{\ln \nu}$. These conditions on $\theta$ are summarized in the table below.

\begin{tabular}{|c|c|c|c|c|}
\hline$r$ & $-\infty$ & $\frac{-1+\alpha}{1+\alpha}$ & 0 & $+\infty$ \\
\hline$\theta$ & $\theta \leq \frac{\ln \alpha}{\ln \nu}$ & $\theta \leq \frac{\ln (1+(1+\alpha) r)}{\ln \nu}$ & $\frac{\ln (1+(1+\alpha) r)}{\ln \nu} \leq \theta \leq \frac{\ln \alpha}{\ln \nu}$ & \\
\hline
\end{tabular}

If $\alpha=0$, we have the same results replacing $\ln \alpha$ by $-\infty$.

Let us now consider the right inequality in $(27): \frac{1-\nu^{\theta(r, \nu)}}{r}+\nu^{\theta\left(r^{\prime}, \nu\right)} \leq \frac{2}{\nu}-1$. We consider a parameter $\beta$ in $[0 ; 1]$. We are looking for the conditions on $\theta$ such that

$$
\left\{\begin{array}{l}
\nu^{\theta\left(r^{\prime}, \nu\right)} \leq \beta\left(\frac{2}{\nu}-1\right) \\
\frac{1-\nu^{\theta(r, \nu)}}{r} \leq(1-\beta)\left(\frac{2}{\nu}-1\right)
\end{array}\right.
$$

After calculus, we obtain the following array:

\begin{tabular}{|c|c|c|c|}
\hline$r$ & 0 & $\frac{1}{\left(\frac{2}{\alpha}-1\right)(1-\beta)}$ & $+\infty$ \\
\hline$\theta$ & $\frac{\ln \beta\left(\frac{2}{\nu}-1\right)}{\ln \nu} \leq \theta \leq \frac{\ln \left(1-\left(\frac{2}{\nu}-1\right)(1-\beta) r\right)}{\ln \nu}$ & & \\
\hline
\end{tabular}

\begin{tabular}{|l|l|l|}
\hline$r$ & $-\infty$ & $\frac{1-\beta\left(\frac{2}{\nu}-1\right)}{(1-\beta)\left(\frac{2}{\nu}-1\right)}$ \\
\hline$\theta$ & $\theta \geq \frac{\ln \beta\left(\frac{2}{\nu}-1\right)}{\ln \nu}$ & $\theta \geq \frac{\ln \left(1-\left(\frac{2}{\nu}-1\right)(1-\beta) r\right.}{\ln \nu}$ \\
\hline
\end{tabular}

Let us remark that if $r \in\left[0 ; \frac{1}{\left(\frac{2}{\nu}-1\right)(1-\beta)}\right]$, the existence of the function $\theta$ implies that

$$
\frac{\ln \beta\left(\frac{2}{\nu}-1\right)}{\ln \nu} \leq \theta(r, \nu) \leq \frac{\ln \left(1-\left(\frac{2}{\nu}-1\right)(1-\beta) r\right)}{\ln \nu}
$$

that is

$$
\beta \geq \frac{1}{\frac{2}{\nu}-1}
$$

\subsubsection{Optimal parameter region}

It appears that several regions are admissible according to the different choices of the two parameters $\alpha$ and $\beta$. We want to identify a region which provides stability and TVD properties with minimal numerical dissipation. According to the equivalent equation, we have to find a stable and TVD region where the function $\theta$ takes maximal value. The conditions on $\alpha$ do not allow us to determine an "optimal" region. To simplify, we decide to take $\alpha=0$.

To choose $\beta$, we fix a real $r>0$. We want to find a parameter $\beta$ which gives the maximal value for 


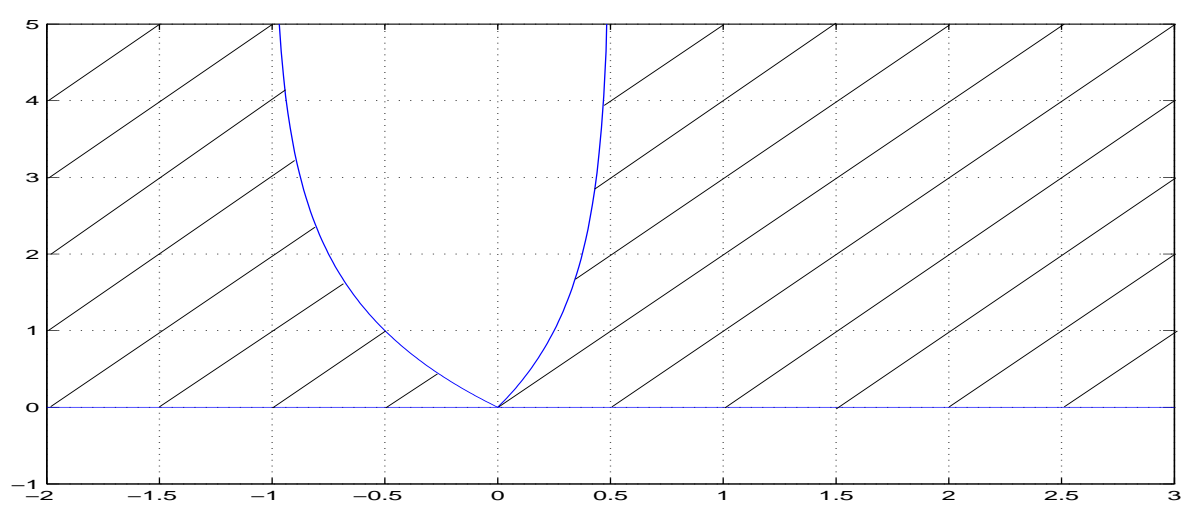

FIGURE 3. TVD and $L^{\infty}$-stablility region (streaked region) in the case $\alpha=0$ and $\beta=\frac{1}{\frac{2}{\nu}-1}$, for $\theta(r)$. The CFL condition is $\nu=0.5$.

$\frac{\ln \left(1-\left(\frac{2}{\nu}-1\right)(1-\beta) r\right)}{\ln \nu}$. As this function of $\beta$ (for a fixed $r$ ) is decreasing, it is sufficient to set $\beta=\frac{1}{\frac{2}{\nu}-1}$. We summarize the new conditions.

Proposition 2.1. Consider a function $\theta$ which satisfies the conditions below.

\begin{tabular}{|c|c|c|c|c|}
\hline$r$ & $-\infty$ & \multicolumn{2}{|c}{-1} & $\frac{1}{2\left(\frac{1}{\nu}-1\right)}$ \\
\hline$\theta$ & $\theta \geq 0$ & $0 \leq \theta \leq \frac{\ln (1+r)}{\ln \nu}$ & $0 \leq \theta \leq \frac{\ln \left(1-2\left(\frac{1}{\nu}-1\right) r\right)}{\ln \nu}$ & $\theta \geq 0$ \\
\hline
\end{tabular}

Then the hybrid scheme (22)-(23) in the linear case has the TVD and the $L^{\infty}$-stability properties.

Let us remark that the proposition 2.1 is an example of sufficient conditions for the function $\theta$. The admissible region for $\theta$ as a function of $r$ is represented in Figure 3.

\subsection{Nonlinear case}

We now consider the nonlinear scalar conservation law:

$$
\partial_{t} u+\partial_{x} f(u)=0
$$

We use the scheme:

with

$$
u_{j}^{n+1}=u_{j}^{n}-\lambda\left(\phi_{j+\frac{1}{2}}^{n}-\phi_{j-\frac{1}{2}}^{n}\right)
$$

$$
\phi_{j+\frac{1}{2}}^{n}=\frac{f\left(u_{j+1}^{n}\right)+f\left(u_{j}^{n}\right)}{2}-\frac{1}{2} \lambda^{\theta_{j+\frac{1}{2}}^{n}}\left|a_{j+\frac{1}{2}}\right|^{1+\theta_{j+\frac{1}{2}}^{n}}\left(u_{j+1}^{n}-u_{j}^{n}\right),
$$

and $a_{j+\frac{1}{2}}=a\left(u_{j}^{n}, u_{j+1}^{n}\right)$ and $(u, v) \mapsto a(u, v)$ the function defined by

$$
a(u, v)= \begin{cases}f^{\prime}(u) & \text { if } u=v \\ \frac{f(v)-f(u)}{v-u} & \text { otherwise. }\end{cases}
$$

We recall that $\Delta u_{j+\frac{1}{2}}=u_{j+1}^{n}-u_{j}^{n}$. Let us assume that there is a constant $\mu$ (independant of $j$ and $n$ ) such that $\left|\lambda f^{\prime}(u)\right| \leq \mu$. To find the TVD and stability region, we follow ideas from flux limiters theory [6,13] as in the linear case. 


\subsubsection{Incremental form of the hybrid scheme}

Thus the scheme (32)-(33)

$$
\begin{aligned}
u_{j}^{n+1}=u_{j}^{n}-\lambda & \left(\frac{f_{j+1}^{n}+f_{j}^{n}}{2}-\frac{1}{2} \lambda^{\theta_{j+\frac{1}{2}}}\left|a_{j+\frac{1}{2}}\right|^{1+\theta_{j+\frac{1}{2}}^{n}} \Delta u_{j+\frac{1}{2}}^{n}\right. \\
& \left.-\left(\frac{f_{j}^{n}+f_{j-1}^{n}}{2}-\frac{1}{2} \lambda^{\theta_{j-\frac{1}{2}}}\left|a_{j-\frac{1}{2}}\right|^{1+\theta_{j-\frac{1}{2}}^{n}} \Delta u_{j-\frac{1}{2}}^{n}\right)\right)
\end{aligned}
$$

can be rewritten as

$$
\begin{aligned}
u_{j}^{n+1}=u_{j}^{n}-\frac{1}{2}\left(\left(\lambda a_{j+\frac{1}{2}}-\left|\lambda a_{j+\frac{1}{2}}\right|^{1+\theta_{j+\frac{1}{2}}^{n}}\right) \Delta u_{j+\frac{1}{2}}^{n}\right. \\
\left.+\left(\lambda a_{j-\frac{1}{2}}+\left|\lambda a_{j-\frac{1}{2}}\right|^{1+\theta_{j-\frac{1}{2}}^{n}}\right) \Delta u_{j-\frac{1}{2}}^{n}\right) .
\end{aligned}
$$

We define $a^{+}$the positive part of $a$ and $a^{-}$the negative part of $a$, that is $a=a^{+}+a^{-}$and $|a|=a^{+}-a^{-}$. We have

$$
\begin{aligned}
u_{j}^{n+1}=u_{j}^{n}-\frac{1}{2}\left(\lambda a_{j+\frac{1}{2}}^{+}\right. & \left(1-\left|\lambda a_{j+\frac{1}{2}}\right|^{\theta_{j+\frac{1}{2}}^{n}}\right) \Delta u_{j+\frac{1}{2}}^{n} \\
& +\lambda a_{j+\frac{1}{2}}^{-}\left(1+\left|\lambda a_{j+\frac{1}{2}}\right|^{\theta_{j+\frac{1}{2}}^{n}}\right) \Delta u_{j+\frac{1}{2}}^{n} \\
& +\lambda a_{j-\frac{1}{2}}^{+}\left(1+\left|\lambda a_{j-\frac{1}{2}}\right|^{\theta_{j-\frac{1}{2}}^{n}}\right) \Delta u_{j-\frac{1}{2}}^{n} \\
& \left.\left.+\lambda a_{j-\frac{1}{2}}^{-}\left(1-\left|\lambda a_{j-\frac{1}{2}}\right|^{\theta^{n}}{ }^{n} \frac{1}{2}\right) \Delta u_{j-\frac{1}{2}}^{n}\right)\right) .
\end{aligned}
$$

We now assume that $\left(a_{j-\frac{1}{2}}^{+} \neq 0\right.$ or $\left.a_{j+\frac{1}{2}}^{+}=0\right)$. This means that it is impossible to have simultaneously: $\left(a_{j-\frac{1}{2}}^{+}=0\right.$ and $\left.a_{j+\frac{1}{2}}^{+} \neq 0\right)$. This assumption is verified in particular if the cell $j$ is not in a neighborhood of a sonic point [5]. To simplify, we assume that we are not near a sonic point.

As in the linear case, we set $\nu_{j}=\lambda a_{j}, \nu_{j}^{+}=\lambda a_{j}^{+}$and $\nu_{j}^{-}=\lambda a_{j}^{-}$. Thus,

$$
\begin{aligned}
u_{j}^{n+1}=u_{j}^{n} & -\frac{\nu_{j-\frac{1}{2}}^{+}}{2}\left(\left(1-\left|\nu_{j+\frac{1}{2}}\right|^{\theta_{j+\frac{1}{2}}^{n}}\right) \frac{\nu_{j+\frac{1}{2}}^{+} \Delta u_{j+\frac{1}{2}}^{n}}{\nu_{j-\frac{1}{2}}^{+} \Delta u_{j-\frac{1}{2}}^{n}}+\left(1+\left|\nu_{j-\frac{1}{2}}\right|^{\theta_{j-\frac{1}{2}}^{n}}\right)\right) \Delta u_{j-\frac{1}{2}}^{n} \\
+ & \frac{-\nu_{j+\frac{1}{2}}^{-}}{2}\left(\left(1+\left|\nu_{j+\frac{1}{2}}\right|^{\theta_{j+\frac{1}{2}}^{n}}\right)+\left(1-\left|\nu_{j-\frac{1}{2}}\right|^{\theta_{j-\frac{1}{2}}^{n}}\right) \frac{\nu_{j-\frac{1}{2}}^{-} \Delta u_{j-\frac{1}{2}}^{n}}{\nu_{j+\frac{1}{2}}^{-} \Delta u_{j+\frac{1}{2}}^{n}}\right) \Delta u_{j+\frac{1}{2}}^{n}
\end{aligned}
$$

with the convention $\frac{\nu_{j+\frac{1}{2}}^{+}}{\nu_{j-\frac{1}{2}}^{+}}=0$ and $\frac{\nu_{j-\frac{1}{2}}^{-}}{\nu_{j+\frac{1}{2}}^{-}}=0$ as soon as the numerator is equal to zero. Then we have the proposition:

Proposition 2.2. Without sonic point, the scheme (32) can be written in incremental form

$$
u_{j}^{n+1}=u_{j}^{n}+C_{j+\frac{1}{2}}^{n} \Delta u_{j+\frac{1}{2}}^{n}-D_{j-\frac{1}{2}}^{n} \Delta u_{j-\frac{1}{2}}^{n},
$$


with the coefficients

$$
\begin{aligned}
D_{j-\frac{1}{2}}^{n} & =\frac{\nu_{j-\frac{1}{2}}^{+}}{2}\left(\left(1-\left|\nu_{j+\frac{1}{2}}\right|^{\theta_{j+\frac{1}{2}}^{n}}\right) \frac{\nu_{j+\frac{1}{2}}^{+} \Delta u_{j+\frac{1}{2}}^{n}}{\nu_{j-\frac{1}{2}}^{+} \Delta u_{j-\frac{1}{2}}^{n}}+\left(1+\left|\nu_{j-\frac{1}{2}}\right|^{\theta_{j-\frac{1}{2}}^{n}}\right)\right), \\
C_{j+\frac{1}{2}}^{n}= & \frac{-\nu_{j+\frac{1}{2}}^{-}}{2}\left(\left(1+\left|\nu_{j+\frac{1}{2}}\right|^{\theta_{j+\frac{1}{2}}^{n}}\right)+\left(1-\left|\nu_{j-\frac{1}{2}}\right|^{\theta_{j-\frac{1}{2}}^{n}}\right) \frac{\nu_{j-\frac{1}{2}}^{-} \Delta u_{j-\frac{1}{2}}^{n}}{\nu_{j+\frac{1}{2}}^{-} \Delta u_{j+\frac{1}{2}}^{n}}\right)
\end{aligned}
$$

where $\nu_{j+\frac{1}{2}}=\lambda a_{j+\frac{1}{2}}$ and $\nu_{j-\frac{1}{2}}=\lambda a_{j-\frac{1}{2}}$.

In the following notation, we omit the time dependency. We then set

$$
r_{j}^{+}=\frac{\nu_{j-\frac{1}{2}}^{+} \Delta u_{j-\frac{1}{2}}^{n}}{\nu_{j+\frac{1}{2}}^{+} \Delta u_{j+\frac{1}{2}}^{n}}, r_{j}^{-}=\frac{\nu_{j+\frac{1}{2}}^{-} \Delta u_{j+\frac{1}{2}}^{n}}{\nu_{j-\frac{1}{2}}^{-} \Delta u_{j-\frac{1}{2}}^{n}} .
$$

We define $\theta_{j+\frac{1}{2}}$ as

$$
\theta_{j+\frac{1}{2}}=\left\{\begin{array}{lll}
\theta\left(r_{j}^{+},\left|\nu_{j+\frac{1}{2}}\right|\right) & \text { if } & \nu_{j+\frac{1}{2}} \geq 0 \\
\theta\left(r_{j+1}^{-},\left|\nu_{j+\frac{1}{2}}\right|\right) & \text { if } & \nu_{j+\frac{1}{2}}<0
\end{array}\right.
$$

where $\theta$ is a function on which we will impose some conditions such that the scheme has the TVD and $L^{\infty}$ stability properties thanks to Harten's criteria. Then we have

$$
\begin{aligned}
D_{j+\frac{1}{2}} & =\frac{\nu_{j+\frac{1}{2}}^{+}}{2}\left(\frac{1-\left|\nu_{j+\frac{3}{2}}\right|^{\theta\left(r_{j+1}^{+}\right)}}{r_{j+1}^{+}}+1+\left|\nu_{j+\frac{1}{2}}\right|^{\theta\left(r_{j}^{+}\right)}\right), \\
C_{j+\frac{1}{2}} & =\frac{-\nu_{j+\frac{1}{2}}^{-}}{2}\left(1+\left|\nu_{j+\frac{1}{2}}\right|^{\theta\left(r_{j+1}^{-}\right)}+\frac{1-\left|\nu_{j-\frac{1}{2}}\right|^{\theta\left(r_{j}^{-}\right)}}{r_{j}^{-}}\right) .
\end{aligned}
$$

\subsubsection{Determination of the TVD and stability region}

We want to find a function $\theta(r, \nu)$ such that $C_{j+\frac{1}{2}}$ and $D_{j+\frac{1}{2}}$ satisfy the conditions:

$$
D_{j+\frac{1}{2}} \geq 0, C_{j+\frac{1}{2}} \geq 0, C_{j+\frac{1}{2}}+D_{j+\frac{1}{2}} \leq 1
$$

for the TVD region and the condition

$$
C_{j+\frac{1}{2}}+D_{j-\frac{1}{2}} \leq 1
$$

\begin{tabular}{|c|c|c|}
\hline$r$ & $-\infty$ & -1 \\
\hline$\theta$ & $0 \leq \theta$ & $0 \leq \theta \leq \frac{\ln (1+r)}{\ln 2}$ \\
\hline
\end{tabular}

for the scheme to be $L^{\infty}$-stable.

Far from a sonic point (in fact, it is sufficient to have the same sign for $a_{j+\frac{1}{2}}, a_{j-\frac{1}{2}}, a_{j+\frac{3}{2}}$ ), we have in particular either $\left(D_{j+\frac{1}{2}}=0\right.$ and $\left.D_{j-\frac{1}{2}}=0\right)$ or $\left(C_{j+\frac{1}{2}}=0\right)$. We give in the following proposition an example of the sufficient conditions for the TVD and stability properties.

Proposition 2.3. Let $\mu$ be the CFL number. Let a function $\theta(r, \nu)$ which satisfies the following conditions:

\begin{tabular}{|l|l|ll|}
\hline$r$ & 0 & $\frac{1}{2\left(\frac{1}{\mu}-1\right)}$ & $+\infty$ \\
\hline$\theta$ & $0 \leq \theta \leq \frac{\ln \left(1-2\left(\frac{1}{\mu}-1\right) r\right)}{\ln \nu}$ & $0 \leq \theta$ & \\
\hline
\end{tabular}


then the hybrid scheme (32)-(33) with the notations (36) and the definition of $\theta_{j+\frac{1}{2}}$ given by (37) has the TVD and $L^{\infty}$-stability properties.

The proof is rather technical. The interested reader will find it in the appendix A.

\subsection{Conditions on $\theta$ to have both space and time second order accuracy (scalar nonlinear case)}

In this section, $(u, v) \mapsto a(u, v)$ is a function such that $a(u, u)=f^{\prime}(u)$. Thus $\left(\partial_{u} a\right)(u, u)=\left(\partial_{v} a\right)(u, u)=$ $\frac{1}{2} f^{\prime \prime}(u)$. We consider a smooth solution $u$ of the equation (1) and we consider a Taylor expansion near the point $\left(x_{j}, t^{n}\right)$.

We use the usual notations: $u_{j}^{n} \simeq u\left(x_{j}, t^{n}\right)$ and $u_{j}^{n+1} \simeq u\left(x_{j}, t^{n+1}\right)$. The partial derivative of $\theta$ with respect to $\nu$ and $r$ are respectively denoted by $\theta_{\nu}$ and $\theta_{r}$. We have the following proposition.

Proposition 2.4. Let $\theta$ a function on $\mathbb{R} \times[0 ; 1[$ such that for all real $\nu$,

$$
\begin{gathered}
\theta(1, \nu)=1, \\
\theta_{r}(1, \nu)=0, \\
\theta_{\nu}(1, \nu)=0 .
\end{gathered}
$$

Then the hybrid scheme (32)-(33) with the notations (36) and the definition of $\theta_{j+\frac{1}{2}}$ given by (37) is a time and space second order scheme.

Again, the interested reader will find the proof in appendix B. We will see in the next section that the conditions (42)-(44) for all $\nu$ are not very difficult to satisfy.

\subsection{Practical examples of functions $\theta$}

We remark that the point of coordinates $(1,1)$ in the plane $(r, \theta)$ has to belong to the curve of $\theta$ when $\nu$ is fixed to obtain the second order property. Then, this point has to belong to the TVD region. We give an example of a two-parameter family $\left(\theta_{p, n}\right)_{p, n}$ which satisfies the conditions (42)-(44) for a CFL number $\mu$ less than $\frac{2}{3}$, for all $p \in \mathbb{N}^{*}$ and $n \in \mathbb{N}$.

$$
\theta_{p, n}(r, \nu)= \begin{cases}0 & \text { if } r \leq 0, \\ \frac{\ln (1-\gamma r)}{\ln (\nu)} & \text { if } 0<r \leq \frac{\left(1-\nu^{p}\right)}{\gamma}, \\ \frac{p-1}{\left(\frac{1-\nu^{p}}{\gamma-1}\right)^{n}}(r-1)^{n}+1 & \text { if } \frac{\left(1-\nu^{p}\right)}{\gamma} \leq r<2-\frac{1-\nu^{p}}{\gamma}, \\ p & \text { if } r \geq 2-\frac{1-\nu^{p}}{\gamma},\end{cases}
$$

where $\gamma=2\left(\frac{1}{\mu}-1\right)$. On figure 4 we have represented the curve of $\theta_{4,4}$ in the case $p=4, n=4, \mu=\nu=0.5$.

\subsection{Hybrid scheme extension for systems of conservation laws}

We heuristically extend our hybrid scheme to the systems. We assume that $\mathbf{A}(\mathbf{u}, \mathbf{v})$ is diagonalisable and we use the notations (17) and (18) of section 1.2. We propose the following scheme

$$
\mathbf{u}_{j}^{n+1}=\mathbf{u}_{j}^{n}-\lambda\left(\phi\left(\mathbf{u}_{j}^{n}, \mathbf{u}_{j+1}^{n}, \lambda, \vec{\theta}_{j+\frac{1}{2}}^{n}\right)-\phi\left(\mathbf{u}_{j-1}^{n}, \mathbf{u}_{j}^{n}, \lambda, \vec{\theta}_{j-\frac{1}{2}}^{n}\right)\right),
$$

with

$$
\phi(\mathbf{u}, \mathbf{v}, \lambda, \vec{\theta})=\frac{\mathbf{f}(\mathbf{u})+\mathbf{f}(\mathbf{v})}{2}-\frac{1}{2}(\lambda \mathbf{I})^{\vec{\theta}}|\mathbf{A}(\mathbf{u}, \mathbf{v})|^{\mathbf{e}+\vec{\theta}}(\mathbf{v}-\mathbf{u})
$$




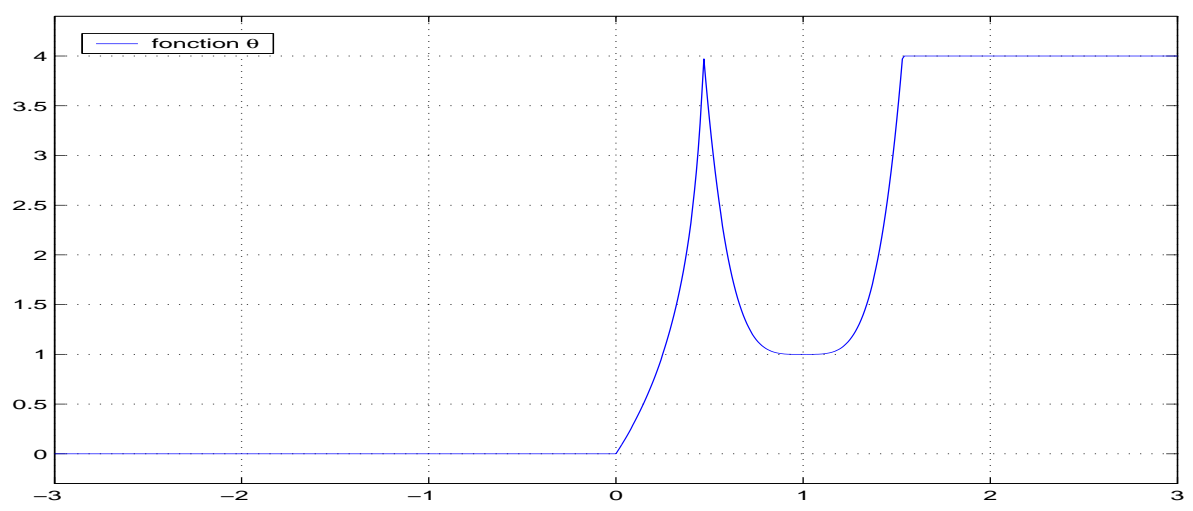

Figure 4 . Function $\theta$ in the case $\mu=0.5, p=4, n=4$.

and $\mathbf{e}=(1, \ldots, 1)$; the matrix $\mathbf{I}$ is the identity matrix in $\mathbb{R}^{p}$. The parameter $\vec{\theta}_{j+\frac{1}{2}}{ }^{n}$ is determined by

$$
\theta_{k, j+\frac{1}{2}}= \begin{cases}\theta\left(r_{k, j}^{+},\left|\nu_{k, j+\frac{1}{2}}\right|\right) & \text { if } \nu_{k, j+\frac{1}{2}} \geq 0 \\ \theta\left(r_{k, j+1}^{-},\left|\nu_{k, j+\frac{1}{2}}\right|\right) & \text { if } \nu_{k, j+\frac{1}{2}}<0\end{cases}
$$

where

$$
\nu_{k, j+\frac{1}{2}}=\lambda \alpha_{k, j+\frac{1}{2}}
$$

and

$$
r_{k, j}^{+}=\frac{\alpha_{k, j-\frac{1}{2}}^{+}\left(\mathbf{R}^{-1}\left(\mathbf{u}_{j}^{n}-\mathbf{u}_{j-1}^{n}\right)\right)_{k}}{\alpha_{k, j+\frac{1}{2}}^{+}\left(\mathbf{R}^{-1}\left(\mathbf{u}_{j+1}^{n}-\mathbf{u}_{j}^{n}\right)\right)_{k}}, r_{k, j}^{-}=\frac{\alpha_{k, j+\frac{1}{2}}^{-}\left(\mathbf{R}^{-1}\left(\mathbf{u}_{j+1}^{n}-\mathbf{u}_{j}^{n}\right)\right)_{k}}{\alpha_{k, j-\frac{1}{2}}^{-}\left(\mathbf{R}^{-1}\left(\mathbf{u}_{j}^{n}-\mathbf{u}_{j-1}^{n}\right)\right)_{k}} .
$$

Here the function $\theta$ is the same as in the scalar case.

\section{NumERICAL EXPERIMENTS}

For numerical experiments, the function $\theta_{4,4}$ represented in Figure 4 is used.

\subsection{Scalar case}

\subsubsection{Linear advection equation with a smooth initial data}

We here consider the linear advection equation:

$$
\left.\partial_{t} u+\partial_{x} u=0, x \in\right] 0 ; 1[, t>0,
$$

with the $\mathcal{C}^{\infty}$ initial data $\left.u(x, 0)=\sin \left(\pi x+\frac{\pi}{4}\right), x \in\right] 0 ; 1[$ and periodical boundary conditions $u(0, t)=\sin (-\pi t+$ $\left.\frac{\pi}{4}\right), t>0$. The analytical solution of that problem is clearly $u(x, t)=\sin \left(\pi(x-t)+\frac{\pi}{4}\right)$. We shall compare the accuracy of the three schemes: hybrid, Roe (TVD but with diffusion) and Lax-Wendroff (not TVD but second order accurate).

We use a uniform mesh grid made of 200 up to 1600 cells. We give the relative error (with the norm $L^{1}$ ) with regard to the space step $h$ in a logarithmic scale. The slopes of $\log _{10}$ err with regard to $\log _{10} h$ is 1.00 for Roe scheme, 1.99 for Lax-Wendroff scheme and 2.01 for the hybrid scheme. We may remark that the hybrid scheme is a second order scheme. 


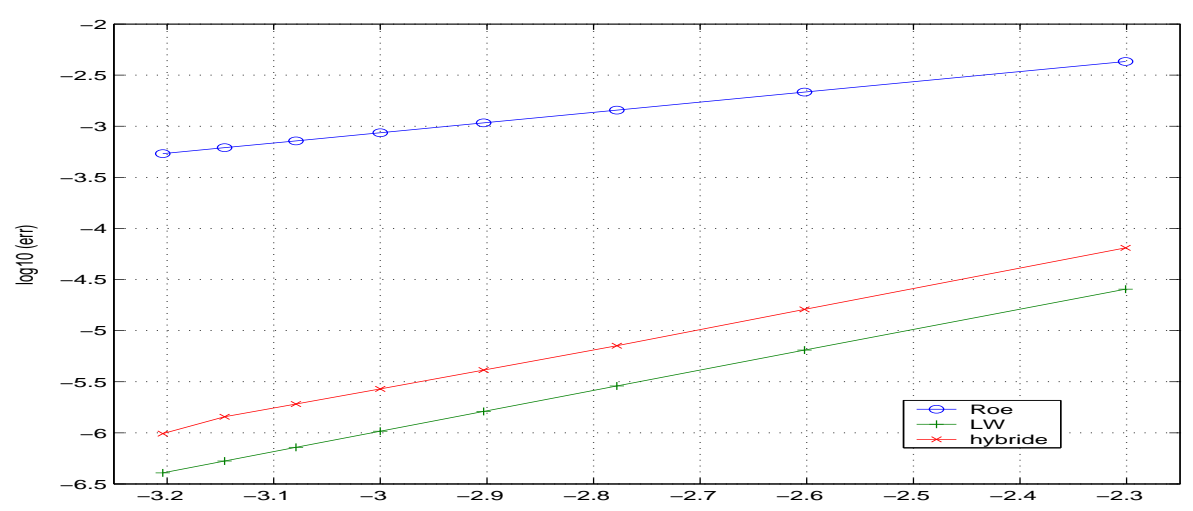

Figure 5. Error for Roe scheme, Lax-Wendroff scheme and hybrid scheme in the case of the linear advection equation with smooth initial data; $\mu=0.5 ; T=0.375$ : curves $\log _{10}$ err with regard to $\log _{10} h$. Slopes: 1.00 for Roe scheme, 1.99 for Lax Wendroff scheme, 2.01 hybrid scheme.

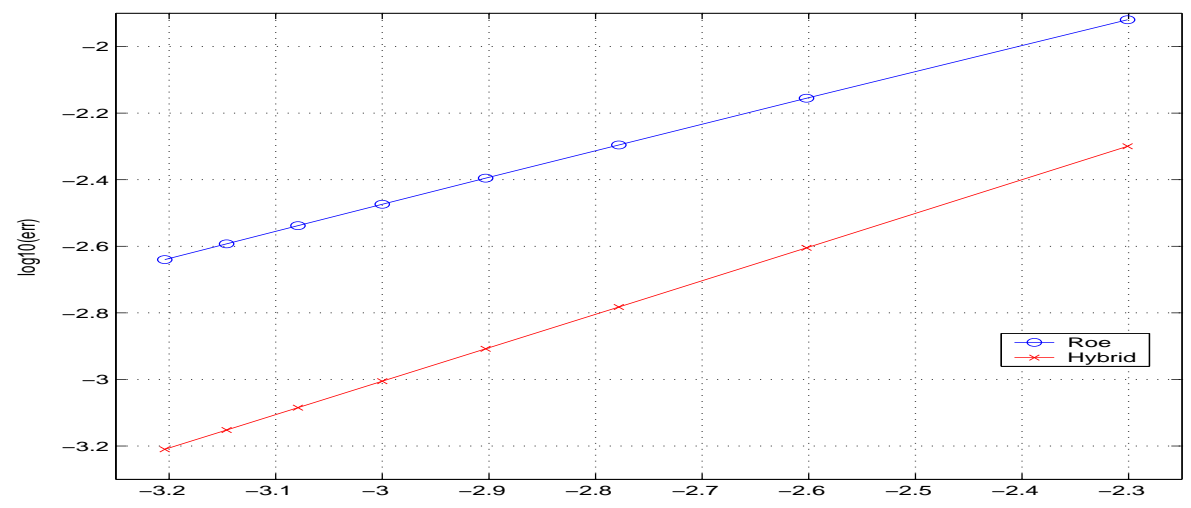

FiguRE 6. Error for Roe scheme and hybrid scheme in the case of the Burger equation with a discontinuous initial data; $\mu=0.5 ; T=0.5$ : curves $\log _{10}$ err with regard to $\log _{10} h$. Slopes: 0.80 for Roe scheme, 1.01 hybrid scheme.

\subsubsection{Riemann problem for Burgers equation}

Let us now consider the inviscid Burgers equation:

$$
\left.\partial_{t} u+\partial_{x}\left(\frac{u^{2}}{2}\right)=0, x \in\right] 0 ; 1[, t>0,
$$

with initial data

$$
u(x, 0)=\left\{\begin{array}{l}
0, \text { if } x \in] 0 ; \frac{1}{5}[, \\
1, \text { if } x \in] \frac{1}{5}, 1[.
\end{array}\right.
$$

We use a uniform mesh grid with 200 to 1600 cells. Figure 6 plots the relative error (with the norm $L^{1}$ ) with regard to the space step $h$ in logarithmic scale. The slopes of $\log _{10} e r r$ with regard to $\log _{10} h$ is 0.80 for Roe scheme and 1.01 for the hybrid scheme. Figures 7 and 8 represent the solution for the Roe scheme and for the hybrid scheme respectively. We use here a uniform meshgrid with 200 points (only 50 points are represented on the figures) and the Courant number is 0.5 . The hybrid scheme gives more accurate results than Roe one. 


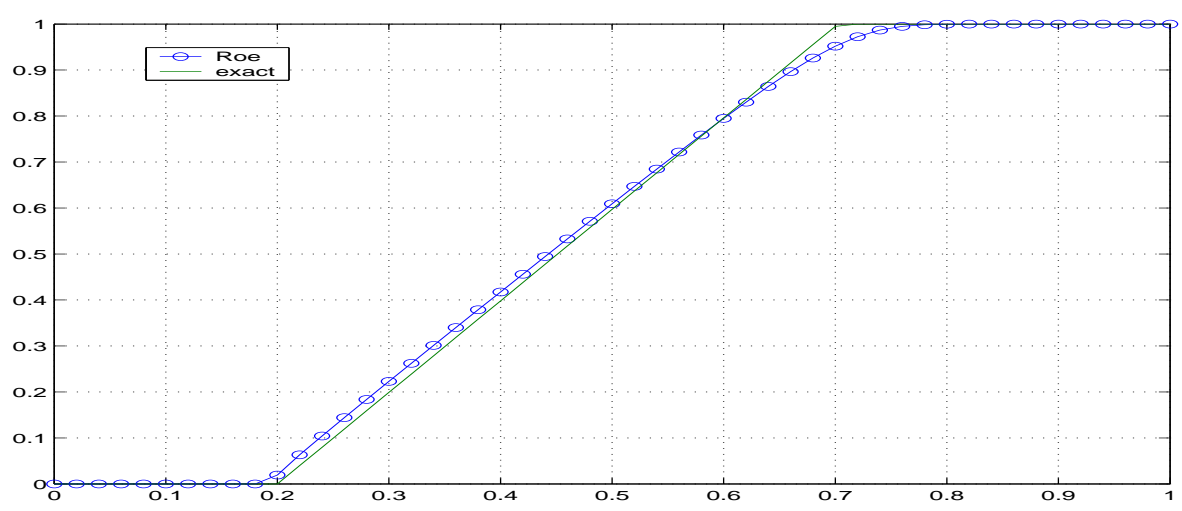

FIGURE 7. Roe scheme and analytical solution for the Burgers equation with a discontinuous initial data; $\mu=0.5 ; T=0.5$.

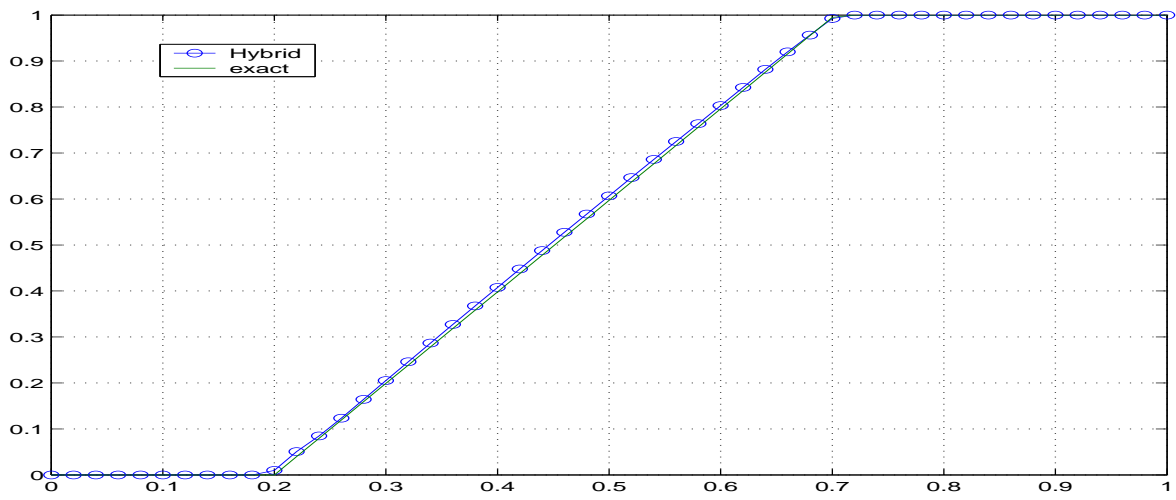

FIGURE 8. Hybrid scheme and analytical solution for the Burgers equation with a discontinuous intial data; $\mu=0.5 ; T=0.5$.

\subsubsection{Burgers equation with a smooth initial data}

Now, we consider the Burgers equation with the $\mathcal{C}^{\infty}$ initial data $\left.u(x, 0)=\sin \left(\pi x+\frac{\pi}{4}\right), x \in\right] 0 ; 1[$ and periodical boundary condition $u(0, t)=\sin \left(-\pi t+\frac{\pi}{4}\right), t>0$. We use a uniform mesh made of 200 points and the Courant Number is 0.5 . The Roe and hybrid schemes (figures 9 and 11 respectively) give the same results except for the beginning of the rarefaction fan which is more accurate for the hybrid scheme. Of course, there are some oscillations for the Lax-Wendroff scheme (figure 10).

\subsection{Numerical experiments for systems of conservation laws}

\subsubsection{Sod shock tube problem for the compressible Euler equations}

Let now consider the compressible Euler equations for the one-dimensional case. The equations are

$$
\left.\partial_{t} \mathbf{u}+\partial_{x} \mathbf{f}(\mathbf{u})=0, x \in\right] 0 ; 1[, t>0
$$

where $\mathbf{u}=(\rho, \rho v, \rho E)$ is the conservative variables vector, $\mathbf{f}(\mathbf{u})=\left(\rho v, \rho v^{2}+p,(\rho E+p) v\right)$ is the flow, $\rho$ the density, $v$ the velocity, $p$ the pressure, $\rho E$ the volumic energy. The assumption of perfect gas gives the equation of state $p=(\gamma-1)\left(\rho E-\frac{1}{2} \rho v^{2}\right)$. We choose $\gamma=1.4$. The Sod shock tube problem [12] consists in a discontinuous piecewise constant initial data made of two constant left and right states $u_{L}=\left(\rho_{L}, \rho_{L} v_{L}, \rho_{L} E_{L}\right)$ and $u_{R}=\left(\rho_{R}, \rho_{R} v_{R}, \rho_{R} E_{R}\right)$ with an interface at $x=0.5$. This corresponds to a Riemann problem. The exact 


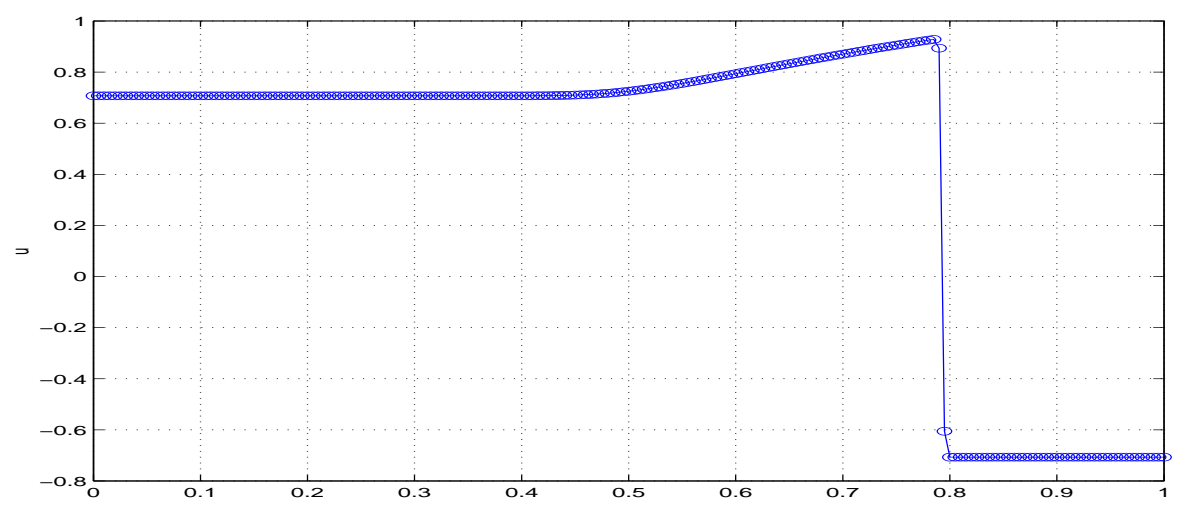

Figure 9. Roe scheme for the Burgers equation with a regular intial data; $\mu=0.5 ; T=0.7$.

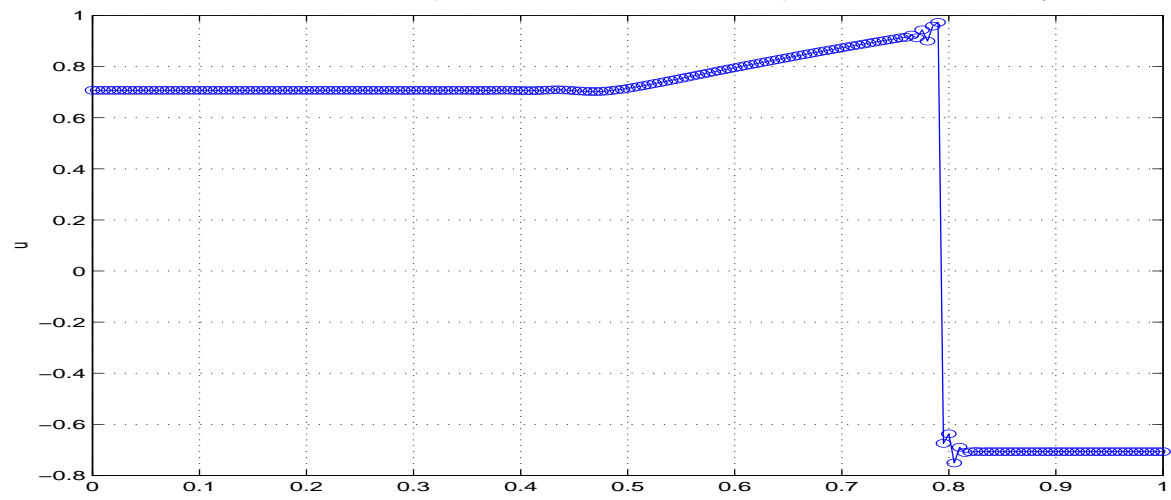

Figure 10. Lax Wendroff scheme for the Burgers equation with a regular intial data; $\mu=0.5$; $T=0.7$.

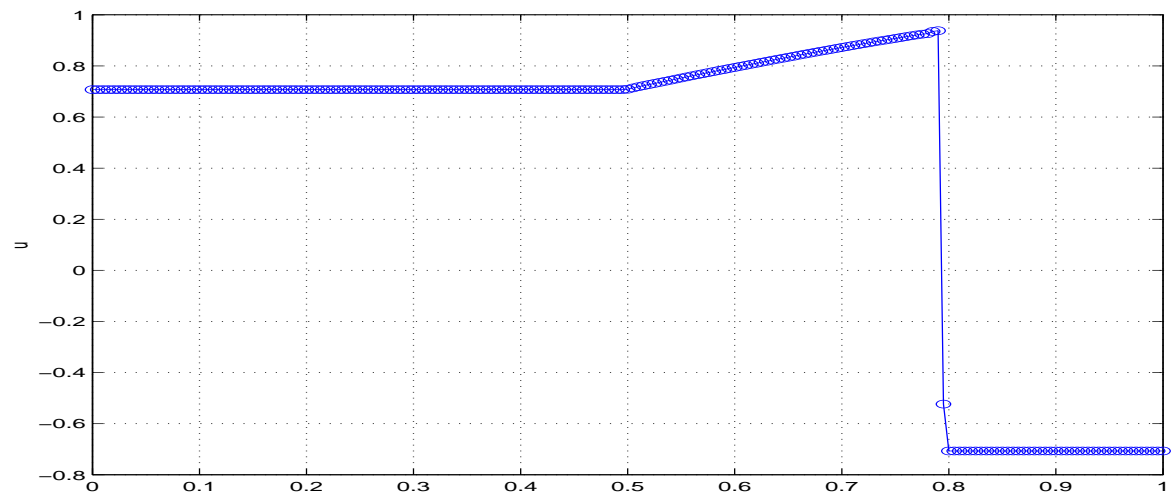

FiguRE 11. Hybrid scheme for the Burgers equation with a regular intial data; $\mu=0.5 ; T=0.7$.

solution is made of a 1-rarefaction, a 2-contact discontinuity and a 3-shock. Initial value are the following: $\left(\rho_{L}, v_{L}, p_{L}\right)=(1,0,1)$ and $\left(\rho_{R}, v_{R}, p_{R}\right)=(0.125,0,0.1)$. We compare Roe and hybrid schemes (figures 12 and 13) with a uniform grid made of 200 points and a Courant number of 0.5. The reference solution (for visual comparison) has been computed using the Roe scheme with Harten entropy fix on a very fine uniform grid (10000 points). The hybrid scheme captures the solution more accurately. 

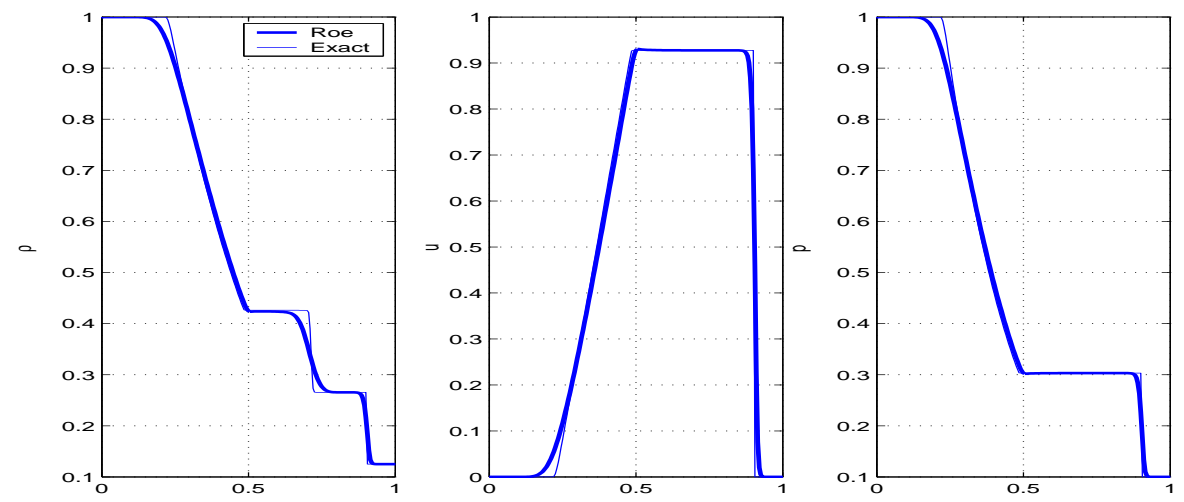

FIGURE 12. Roe scheme and reference solution for the Euler equations: $\rho, u, p$ respectively for Sod shock tube problem, 200 points $\nu=0.5 ; T=0.23$.
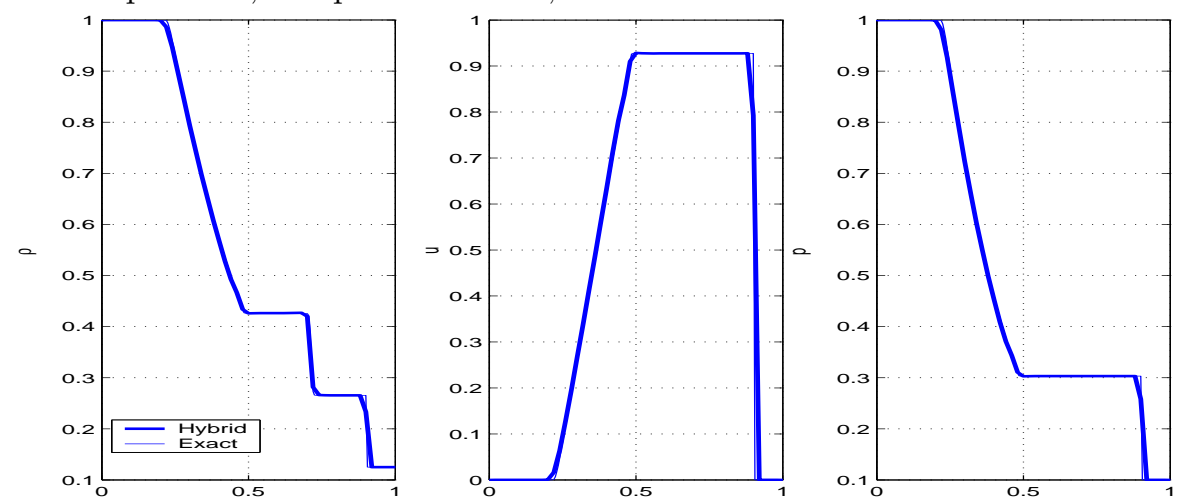

FIGURE 13. Hybrid scheme and reference solution for the Euler equations: $\rho, u, p$ respectively for Sod shock tube problem, 200 points $\nu=0.5 ; T=0.23$.

\subsubsection{Transonic rarefaction fan for the Euler equations}

Let us consider the shock tube problem of initial data $\left(\rho_{L}, v_{L}, p_{L}\right)=(5,0,5)$ and $\left(\rho_{R}, v_{R}, p_{R}\right)=(0.125,0,0.1)$ with an interface at $x=0.5$. We compare the Roe and hybrid scheme (figures 14 and 15) with a uniform grid made of 100 points and a Courant number of 0.45 . The reference curves (for visual comparison) have been computed using the Roe scheme with Harten entropy fix and a very fine uniform grid (10000 points). We see that the hybrid scheme is more accurate than the Roe scheme. Yet, the hybrid scheme (as the Roe scheme) violates the entropy condition at sonic point $(x=0.5)$ and an entropy shock appears. This drawback can be easily avoided thanks to a regularisation of the absolute value in the viscosity matrix (see [7]): we replace $|\mathbf{A}|=\mathbf{R} \operatorname{diag}\left(\left|\alpha_{k}\right|\right) \mathbf{R}^{-1}$ by $|\mathbf{A}|=\mathbf{R} \operatorname{diag}\left(\psi\left(\alpha_{k}\right)\right) \mathbf{R}^{-1}$, where $\psi$ is defined by

$$
\psi(x)= \begin{cases}\frac{x^{2}}{4 \epsilon} & \text { if }|x|<2 \epsilon \\ |x| & \text { otherwise. }\end{cases}
$$

We choose $\epsilon=0.1$. The results are given in figure 16. Another way is to follow ideas from De Vuyst [1]: we may use entropy criteria to increase the diffusion thanks to the parameter $\theta$. 

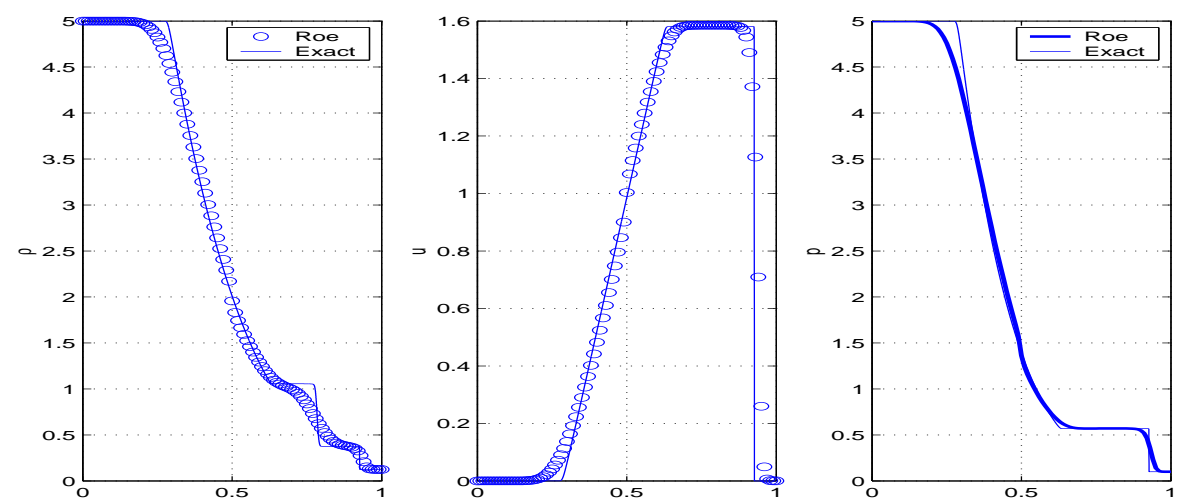

FIGURE 14. Roe scheme and analytical solution for the Euler equations with a rarefaction fan: $\rho, u, p$ respectively; 100 points $\nu=0.45 ; T=0.18$.
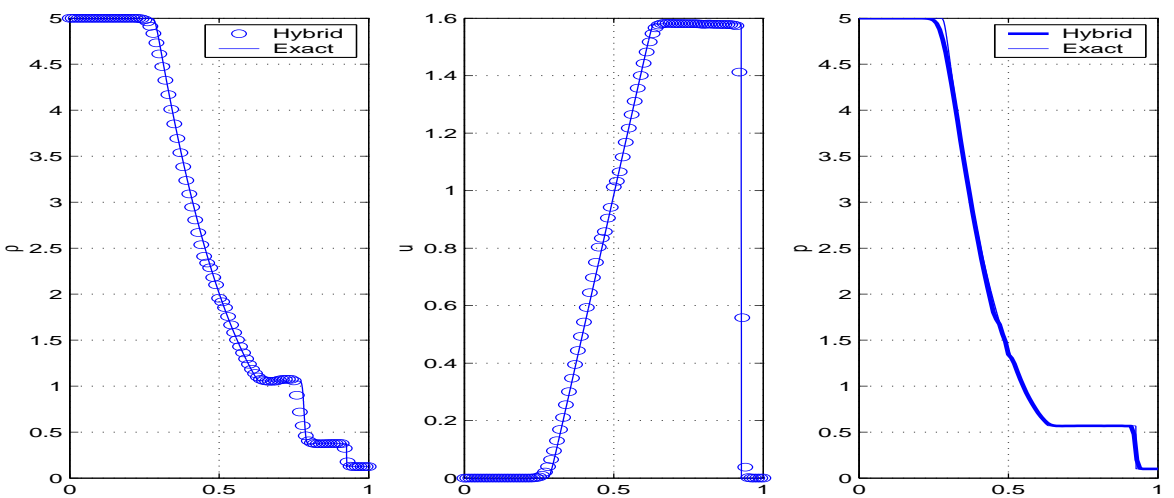

FIGURE 15. Hybrid scheme and analytical solution for the Euler equations with a rarefaction fan: $\rho, u, p$ respectively; 100 points $\nu=0.45 ; T=0.18$.
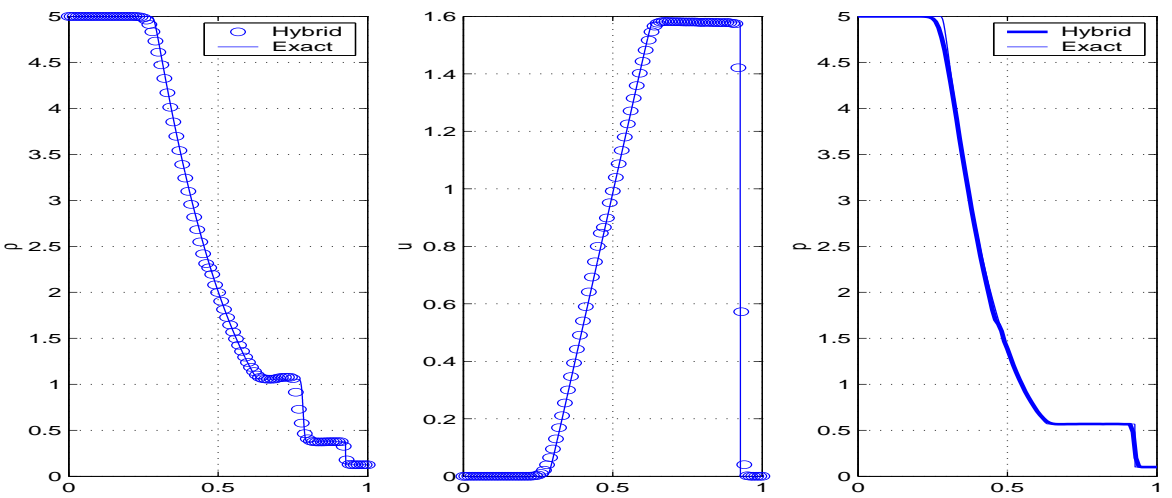

FIGURE 16. Hybrid scheme with regularisation for the Euler equations with a rarefaction fan: $\rho, u, p$ respectively; 100 points $\nu=0.45 ; T=0.18$. 


\section{Concluding Remarks AND ONGOING WORKS}

In this work, we have presented a novel one-parameter hybrid scheme. The parameter can be adjusted in each cell of the meshgrid in order to make the scheme second order accurate, $L^{\infty}$-stable and TVD. The hybrid flux is an interpolation of some classical numerical fluxes (Lax-Wendroff, Roe, Lax-Friedrichs or others) thanks to this parameter. On one hand, a low value of the parameter allows us to get the TVD property in the region where the solution is not smooth or the gradient is very important. On the other hand, the parameter can take a higher value to make the scheme accurate in regions where the solution is smooth. We obtain a TVD second order scheme. This scheme is easy to implement with parameter function in closed form. The hybrid scheme provides very good numerical results and should be very promising for real or complex applications.

We have already extended the hybrid scheme to the multidimensional case using cartesian meshgrids. The numerical results show both strong accuracy with sharp discontinuities.

Sometimes it is necessary to use regularisation in the viscosity matrix to make the scheme entropy-satisfying (as Roe scheme, for example). This correction is very easy to implement.

\section{A. Proof of proposition 2.3}

We are looking for some sufficient conditions to have a TVD and stable scheme.

a) Conditions for $D_{j+\frac{1}{2}} \geq 0$.

We assume $a_{j+\frac{1}{2}}>0$ and then $a_{j-\frac{1}{2}}>0$ and $a_{j+\frac{3}{2}}>0$. Thus far away from a sonic point, $C_{j+\frac{1}{2}}=0$. To simplify, we set $\nu^{\prime}=\nu_{j+\frac{1}{2}}^{+}$and $\nu^{\prime \prime}=\left|\nu_{j+\frac{3}{2}}\right|$. With these notations, we have:

$$
\frac{\nu^{\prime}}{2}\left(1+\nu^{\prime \theta^{\prime}}+\frac{1-\nu^{\prime \prime \theta\left(r^{\prime \prime}, \nu^{\prime \prime}\right)}}{r^{\prime \prime}}\right) \geq 0 .
$$

As $\nu^{\prime \theta^{\prime}} \geq 0$, it is sufficient (but not necessary, see the linear case) to have

$$
\frac{1-\nu^{\prime \prime \theta\left(r^{\prime \prime}, \nu^{\prime \prime}\right)}}{r^{\prime \prime}} \geq-1 \text {. }
$$

This inequality is equivalent to

$$
\begin{cases}\theta\left(r^{\prime \prime}, \nu^{\prime \prime}\right) \geq \frac{\ln \left(1+r^{\prime \prime}\right)}{\ln \nu^{\prime \prime}}, & \text { if } r^{\prime \prime} \geq 0 \\ \theta\left(r^{\prime \prime}, \nu^{\prime \prime}\right) \leq \frac{\ln \left(1+r^{\prime \prime}\right)}{\ln \nu^{\prime \prime}}, & \text { if }-1 \leq r^{\prime \prime}<0\end{cases}
$$

(if $r^{\prime \prime}<-1$, the inequality is always verified).

b) Conditions for $C_{j+\frac{1}{2}} \geq 0$.

If we consider $a_{j+\frac{1}{2}}<0$, we have far away from a sonic point, $D_{j+\frac{1}{2}}=0$. In fact, we obtain the same conditions.

c) Conditions for $C_{j+\frac{1}{2}}+D_{j-\frac{1}{2}} \leq 1$.

For example, we assume $a_{j+\frac{1}{2}}^{-}=0$. Thus

$$
D_{j+\frac{1}{2}} \leq 1
$$

that is

$$
\frac{\nu^{\prime}}{2}\left(\frac{1-\nu^{\prime \prime \theta\left(r^{\prime \prime}, \nu^{\prime \prime}\right)}}{r^{\prime \prime}}+1+\nu^{\prime \theta\left(r^{\prime}, \nu^{\prime}\right)}\right) \leq 1
$$


then

$$
\frac{1-\nu^{\prime \prime \theta\left(r^{\prime \prime}, \nu^{\prime \prime}\right)}}{r^{\prime \prime}}+\nu^{\prime \theta\left(r^{\prime}, \nu^{\prime}\right)} \leq \frac{2}{\nu^{\prime}}-1
$$

As in the linear case, we consider a real parameter $\beta$ in $[0 ; 1]$. We are looking for the functions $\theta$ such that

$$
\left\{\begin{array}{l}
\nu^{\prime \theta\left(r^{\prime}, \nu^{\prime}\right)} \leq \beta\left(\frac{2}{\nu^{\prime}}-1\right) \\
\frac{1-\nu^{\prime \prime \theta\left(r^{\prime \prime}, \nu^{\prime \prime}\right)}}{r^{\prime \prime}} \leq(1-\beta)\left(\frac{2}{\nu^{\prime}}-1\right)
\end{array}\right.
$$

The unique difference from the linear case is for the second inequality $\frac{1-\nu^{\prime \prime \theta\left(r^{\prime \prime}, \nu^{\prime \prime}\right)}}{r^{\prime \prime}} \leq(1-\beta)\left(\frac{2}{\nu^{\prime}}-1\right)$ : we have supposed that $\nu^{\prime} \leq \mu$ thus $\frac{2}{\nu^{\prime}}-1 \geq \frac{2}{\mu}-1$. Then it is sufficient to solve

$$
\frac{1-\nu^{\prime \prime \theta\left(r^{\prime \prime}, \nu^{\prime \prime}\right)}}{r^{\prime \prime}} \leq(1-\beta)\left(\frac{2}{\mu}-1\right) .
$$

If we suppose now that $a_{j+\frac{1}{2}}^{+}=0$, then we can easily prove that there is no new conditions.

\section{d) Summary of the conditions on $\theta$.}

We obtain after calculus a first array which gives the condition on the upper bound of $\theta$.

\begin{tabular}{|l|l|l|}
\hline$r$ & $-\infty$ & $\frac{1-\beta\left(\frac{2}{\nu}-1\right)}{(1-\beta)\left(\frac{2}{\nu}-1\right)}$ \\
\hline$\theta$ & $\theta \geq \frac{\ln \beta\left(\frac{2}{\nu}-1\right)}{\ln \nu}$ & $\theta \geq \frac{\ln \left(1-\left(\frac{2}{\mu}-1\right)(1-\beta) r\right)}{\ln \nu}$ \\
\hline
\end{tabular}

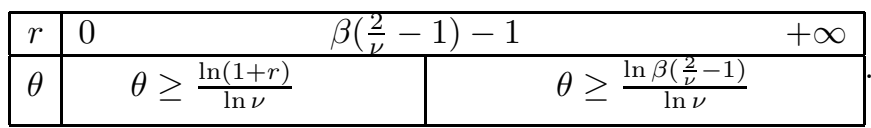

The second array gives the condition on the lower bound of $\theta$.

\begin{tabular}{|l|l|l|l|l|}
\hline$r$ & $-\infty$ & -1 & \multicolumn{3}{|c|}{0} & $\frac{1}{\left(\frac{2}{\mu}-1\right)(1-\beta)} \quad+\infty$ \\
\hline$\theta$ & & $\theta \leq \frac{\ln (1+r)}{\ln \nu}$ & $\theta \leq \frac{\ln \left(1-\left(\frac{2}{\mu}-1\right)(1-\beta) r\right.}{\ln \nu}$ & \\
\hline
\end{tabular}

If $r$ belongs to $\left[0 ; \frac{1}{\left(\frac{2}{\nu}-1\right)(1-\beta)}\right]$, the parameter $\beta$ must satisfy

$$
\frac{\ln \beta\left(\frac{2}{\nu}-1\right)}{\ln \nu} \leq \theta(r, \nu) \leq \frac{\ln \left(1-\left(\frac{2}{\mu}-1\right)(1-\beta) r\right)}{\ln \nu},
$$

and if $r$ belongs to $[-1 ; 0]$, the parameter $\beta$ must satisfy

$$
\frac{\ln (1+r)}{\ln \nu} \leq \theta(r, \nu) \leq \frac{\ln \left(1-\left(\frac{2}{\mu}-1\right)(1-\beta) r\right)}{\ln \nu}
$$

The first condition is always verified and the second condition is equivalent to

$$
\beta \geq \frac{1}{\frac{2}{\nu}-1}
$$


which must be verified for all $\nu$ such that $0 \leq \nu \leq \mu$. Thus

$$
\beta \geq \frac{1}{\frac{2}{\mu}-1}
$$

As in the linear case, we set $\beta=\frac{1}{\frac{2}{\mu}-1}$.

\section{B. Proof of proposition 2.4}

To simplify, we will omit the exponant index $n$ when we will consider $t^{n}$ and the index $j$ when we will consider the value in $x_{j}$. For instance, we note $f\left(u_{j}^{n}\right)$ by $f$ and $f^{\prime}\left(u_{j}^{n}\right)$ by $f_{u}$.

We set

$$
R_{j}^{n}=u_{j}^{n+1}-u_{j}^{n}+\lambda\left(\phi_{j+\frac{1}{2}}^{n}-\phi_{j-\frac{1}{2}}^{n}\right) .
$$

We recall that the numerical flux is given by:

$$
\phi_{j+\frac{1}{2}}^{n}=\frac{f\left(u_{j+1}^{n}\right)+f\left(u_{j}^{n}\right)}{2}-\frac{1}{2} \lambda^{\theta_{j+\frac{1}{2}}^{n}}\left|a_{j+\frac{1}{2}}\right|^{1+\theta_{j+\frac{1}{2}}^{n}}\left(u_{j+1}^{n}-u_{j}^{n}\right) .
$$

First, we suppose that $a_{j+\frac{1}{2}}>0$ and $a_{j-\frac{1}{2}}>0$. We will see that the proof will be the same when one or two quantities are negative. Thus

$$
\theta_{j+\frac{1}{2}}=\theta\left(r_{j}^{+}, \nu_{j+\frac{1}{2}}\right), \theta_{j-\frac{1}{2}}=\theta\left(r_{j-1}^{+}, \nu_{j-\frac{1}{2}}\right)
$$

and

The truncature error is

$$
r_{j}^{+}=\frac{a_{j-\frac{1}{2}}^{+} \Delta u_{j-\frac{1}{2}}^{n}}{a_{j+\frac{1}{2}}^{+} \Delta u_{j+\frac{1}{2}}^{n}} .
$$

$$
\begin{aligned}
R_{j}^{n}=u_{j}^{n+1}-u_{j}^{n}+\lambda( & \frac{f_{j+1}^{n}-f_{j-1}^{n}}{2} \\
& \left.-\frac{1}{2}\left(\lambda^{\theta_{j+\frac{1}{2}}}\left|a_{j+\frac{1}{2}}^{n}\right|^{1+\theta_{j+\frac{1}{2}}} \Delta u_{j+\frac{1}{2}}^{n}-\lambda^{\theta_{j-\frac{1}{2}}}\left|a_{j-\frac{1}{2}}^{n}\right|^{1+\theta_{j-\frac{1}{2}}} \Delta u_{j-\frac{1}{2}}^{n}\right)\right) .
\end{aligned}
$$

We have

$$
\begin{aligned}
& u_{j}^{n+1}-u_{j}^{n}=u_{t} \Delta t+\frac{u_{t t}}{2} \Delta t^{2}+o\left(\Delta t^{2}\right), \\
& \Delta u_{j+\frac{1}{2}}^{n}=u_{j+1}^{n}-u_{j}^{n}=u_{x} h+\frac{u_{x x}}{2} h^{2}+o\left(h^{2}\right), \\
& \Delta u_{j-\frac{1}{2}}^{n}=u_{j}^{n}-u_{j-1}^{n}=u_{x} h-\frac{u_{x x}}{2} h^{2}+o\left(h^{2}\right),
\end{aligned}
$$

and

$$
\begin{aligned}
& a_{j+\frac{1}{2}}^{+}=a_{j+\frac{1}{2}}=a+a_{u} u_{x} h+o(h), \\
& a_{j-\frac{1}{2}}^{+}=a_{j-\frac{1}{2}}=a-a_{u} u_{x} h+o(h) .
\end{aligned}
$$

Then we obtain :

$$
r_{j}^{+}=1-\left(\frac{u_{x x}}{u_{x}}+2 \frac{a_{u}}{a} u_{x}\right) h+o(h)
$$


then

$$
\theta_{j+\frac{1}{2}}=\theta(1, \lambda a)+\left(-\theta_{r}(1, \lambda a)\left(\frac{u_{x x}}{u_{x}}+2 \frac{a_{u}}{a} u_{x}\right)+\theta_{\nu}(1, \lambda a) \lambda a_{u} u_{x}\right) h+o(h)
$$

and

$$
\begin{aligned}
\lambda^{\theta_{j+\frac{1}{2}}}\left|a_{j+\frac{1}{2}}\right|^{1+\theta_{j+\frac{1}{2}}}=\lambda^{\theta(1, \lambda a)} a^{1+\theta(1, \lambda a)}\left(1-\ln (\lambda a)\left(\theta_{r}(1, \lambda a)\left(\frac{u_{x x}}{u_{x}}+2 \frac{a_{u}}{a} u_{x}\right)\right.\right. \\
\left.\left.-\theta_{\nu}(1, \lambda a) \lambda a_{u} u_{x}+(1+\theta(1, \lambda a)) \frac{a_{u}}{a} u_{x}\right) h\right)+o(h) .
\end{aligned}
$$

In the same manner,

$$
\begin{aligned}
\lambda^{\theta_{j-\frac{1}{2}}}\left|a_{j-\frac{1}{2}}\right|^{1+\theta_{j-\frac{1}{2}}}=\lambda^{\theta(1, \lambda a)} a^{1+\theta(1, \lambda a)}(1 & -\ln (\lambda a)\left(\theta_{r}(1, \lambda a)\left(\frac{1}{2} \frac{u_{x x}}{u_{x}}+2 \frac{a_{u}}{a} u_{x}\right)\right. \\
& \left.\left.\left.+\theta_{\nu}(1, \lambda a) \lambda a_{u} u_{x}\right)-(1+\theta(1, \lambda a)) \frac{a_{u}}{a} u_{x}\right) h\right)+o(h) .
\end{aligned}
$$

Moreover

$$
\frac{f_{j+1}^{n}-f_{j-1}^{n}}{2}=a u_{x} h+o\left(h^{2}\right)
$$

Thus,

$$
\begin{aligned}
R= & u_{t} \Delta t+\frac{u_{t t}}{2} \Delta t^{2}+a u_{x} \Delta t \\
& -\frac{1}{2} \Delta t^{\theta(1, \lambda a)+1} h^{-\theta(1, \lambda a)} a^{1+\theta(1, \lambda a)}\left(\ln (\lambda a)\left(\theta_{r}(1, \lambda a) \frac{-u_{x x}}{2}+2 \theta_{\nu}(1, \lambda a) \lambda a_{u} u_{x}\right)\right. \\
& -\frac{1}{2} \Delta t^{\theta(1, \lambda a)+1} h^{1-\theta(1, \lambda a)} a^{1+\theta(1, \lambda a)}\left(2 \frac{a_{u}}{a} u_{x}^{2}(1+\theta(1, \lambda a))+u_{x x}\right) \\
& +o\left(h^{2}\right)+o\left(\Delta t^{2}\right) .
\end{aligned}
$$

But if $u$ is a regular solution of

$$
\partial_{t} u+\partial_{x} f(u)=0
$$

then we have

$$
\left\{\begin{array}{l}
u_{x t}+f^{\prime \prime}(u) u_{x}^{2}+f^{\prime}(u) u_{x x}=0 \\
u_{t t}+f^{\prime \prime}(u) u_{t} u_{x}+f^{\prime}(u) u_{x t}=0 .
\end{array}\right.
$$

By multiplying the first equation by $-f^{\prime}(u)$ and by summing up the two equations, we get, thanks to the relation $u_{t}=-f^{\prime}(u) u_{x}$ :

$$
u_{t t}-\left(f^{\prime}(u)\right)^{2} u_{x x}-2 f^{\prime \prime}(u) f^{\prime}(u) u_{x}^{2}=0 .
$$

But $a=f^{\prime}(u)$ and $a_{u}=\frac{1}{2} f^{\prime \prime}(u)$ then

$$
u_{t t}-a^{2} u_{x x}-4 a_{u} a u_{x}^{2}=0
$$

Then, if we impose for all real $\nu$,

$$
\theta(1, \nu)=1, \theta_{r}(1, \nu)=0, \theta_{\nu}(1, \nu)=0,
$$

we get

$$
R=o\left(h^{2}\right)+o\left(\Delta t^{2}\right) .
$$

Lastly, the assumptions $a_{j+\frac{1}{2}}>0$ and $a_{j-\frac{1}{2}}>0$ appear only in the first order expansion of $\theta$. If we impose $\theta_{r}(1, \lambda a)=0$ and $\theta_{\nu}(1, \lambda a)=0$, the signs of $a_{j+\frac{1}{2}}$ and $a_{j-\frac{1}{2}}$ have no more importance for the proof. 


\section{REFERENCES}

[1] F. DeVuyst. Stable and accurate hybrid finite volume methods based on pure convexity arguments for hyperbolic systems of conservation law. J. Comput. Phys., 193:426-468, 2004.

[2] K.O. Friedrichs. Symmetric hyperbolic linear differential equations. Comm. Pure Appl. Math., 7:345-392, 1954.

[3] J.M. Ghidaglia, A. Kumbaro, and G. Le Coq. Une méthode "volumes finis" à flux caractéristiques pour la résolution numérique des systèmes hyperboliques de lois de conservation. C.R. Acad. Sci. Paris Sér I Math., 322(10):981-988, 1996.

[4] J.M. Ghidaglia, A. Kumbaro, and G. Le Coq. On the numerical solution to two fluid models via a centered finite volume scheme. Europ. J. Mech. B Fluids, 20(6):841-867, 2001.

[5] E. Godlewski and P.A. Raviart. Hyperbolic systems of conservation laws. Paris, Ellipses, 1991.

[6] E. Godlewski and P.A. Raviart. Numerical Approximation of Hyperbolic Systems of Conservation Laws. New York, Springer, 1996.

[7] A. Harten. High resolution schemes for hyperbolic conservation laws. J. of Comput. Phys., 49:357-393, mars 1983.

[8] P.D. Lax. Weak solutions of non-linear hyperbolic equations and their numerical computations. Comm. Pure Appl. Math., 7:159-193, 1954.

[9] P.D. Lax and B. Wendroff. Systems of conservation laws. Commun. Pure Appl. Math., 13:217-237, 1960.

[10] P.L. Roe. Approximate riemann solvers, parameter vector and difference scheme. J. of Comput. Phys, 43:357-372, 1981.

[11] V.V. Rusanov. Calculation of interaction of non-steady shock-waves with obstacles. J. Comput. Math. Phys., 1:267-279, 1961.

[12] G. Sod. A survey of several finite difference methods for systems of non linear hyperbolic conservation laws. J. Comput. Phys., 27:1-31, 1978.

[13] P.K. Sweby. High resolution schemes using flux limiters for hyperbolic conservation laws. SIAM J. Numer. Anal., 21(5):995$1011,1984$.

[14] E. Tadmor. Numerical viscosity and the entropy condition for conservative difference scheme. Math. Comp., 43:369-381, 1984. 